\title{
Numerical identification method for the non-linear \\ viscoelastic compressible behaviour of soft tissue using uniaxial tensile tests and image registration - Application to rat lung parenchyma
}

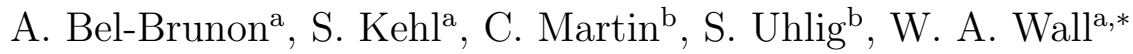 \\ ${ }^{a}$ Institute for Computational Mechanics, Technische Universität München, \\ Boltzmannstrasse 15, 85747 Garching, Germany \\ ${ }^{b}$ Institute of Pharmacology and Toxicology, Medical Faculty, RWTH Aachen University, \\ Aachen, Germany
}

\begin{abstract}
This paper presents an improved identification method of the constitutive properties of lung parenchyma. The aim of this work is to determine the nonlinear viscoelastic behavior of lung parenchyma with a particular focus on the compressible properties. Tensile tests are performed on living precision-cut rat lung slices. Image registration is used to compute the displacement field at the surface of the sample. This allows to identify the compressibility of the tissue. The constitutive model consists of a hyperelastic potential split into volumetric and isochoric contributions and a viscous contribution. This allows the description of the experimentally observed hysteresis loop. The identification is performed numerically: each test is simulated using the real geometry of the sample; the error in the displacement (i.e. the difference between the measured and computed displacements) is minimized with an improved Levenberg-Marquardt algorithm. This global method allows the comparison between several hyperelastic potentials and we can determine
\end{abstract}

\footnotetext{
${ }^{*}$ Corresponding author. Institute for Computational Mechanics, Technische Universität

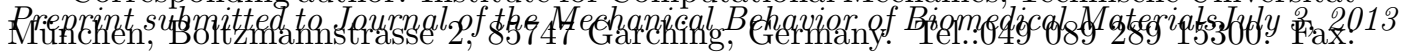
+49089289 15301. E-mail address: wall@lnm.mw.tum.de
} 
the most suitable law for rat lung parenchyma. We find that both an exponential potential or a polynomial potential with a first order term and a third or higher order term give similarly satisfactory results. The identified parameters are: for the volumetric contribution: $\kappa=7.25 \mathrm{e} 4 \pm 2.74 \mathrm{e} 4 \mathrm{~Pa}$, for the exponential potential: $k_{1}=4.70 \mathrm{e} 3 \pm 1.60 \mathrm{e} 3 \mathrm{~Pa}, k_{2}=5.90 \pm 1.51$, for the polynomial form: $C_{1}=2.87 \mathrm{e} 3 \pm 9.05 \mathrm{e} 2 \mathrm{~Pa}, C_{3}=3.83 \mathrm{e} 4 \pm 1.63 \mathrm{e} 4 \mathrm{~Pa}$. The identification of the time parameter for the viscous contribution shows that it depends on the loading frequency $(0.2 \mathrm{~Hz}: \tau=0.257 \pm 0.042 \mathrm{~s}, 0.4$ Hz: $\tau=0.123 \pm 0.039 \mathrm{~s}, 0.8 \mathrm{~Hz}: \tau=0.050 \pm 0.025 \mathrm{~s}$ ). Adding a viscous contribution significantly increases the accuracy of the identification. Keywords: lung parenchyma, non-linear viscoelasticity, numerical identification, image registration, uniaxial tensile test, compressibility

\section{Introduction}

Characterizing the mechanical behavior of lung tissues has been the subject of several scientific studies as it is required to model the global and local behavior of the lung. A computational model of the whole lung could help understanding several phenomena related to lung diseases. For instance, acute respiratory distress syndrome (ARDS) is a disease that alters the mechanical behavior of the lung parenchyma (Kallet and Katz, 2003). When a patient suffering from ARDS is mechanically ventilated to help him breathe, healthy and diseased areas are deformed but the compliance difference of these two tissues can lead to a local overstretching in the parenchyma. A lung model including mechanical properties of both healthy and diseased parenchyma could help locating and quantifying the possible overstretching 
occurring within the parenchyma and adapting the curing protocol to avoid it. A comprehensive model of the lung is currently developed in our group including among others, structure and fluid phenomena (Wall et al., 2010).

Lung parenchyma consists of alveolar tissue, including respiratory bronchioles, alveolar ducts and terminal bronchioles. At the macroscale (a few $\mathrm{cm}$ ), it can be considered as homogeneous but at the microscale (alveolar scale, i.e. a mm), it exhibits a foam-like structure due to the alveoli, which are inflated with air during breathing. Therefore, unlike most of the soft tissues commonly studied (e.g. abdominal organs, skin, aorta), it cannot be considered as incompressible at the macroscale as its main function is to be inflated. Most of the previous studies to characterize the lung parenchyma have investigated its non-linear elasticity and viscoelasticity but the compressible properties have not been characterized sufficently so far.

To characterize lung parenchyma, the few available previous studies describe mostly uniaxial tensile tests on tissue slices. Briefly, in terms of elasticity, either an exponential potential (Navajas et al., 1995) or a polynomial potential (Rausch et al., 2011) describe rather accurately the uniaxial behavior of the lung parenchyma. Gao et al. performed biaxial tensile tests and concluded that an exponential law was the best fit for the tissue behavior (Gao et al., 2006). A volumetric component of the constitutive law is only assessed in (Rausch et al., 2011); however the method to identify the volumetric parameters of the constitutive law only relied on the displacement of a single location in the sample and the measurement of this displacement was done by hand.

In terms of viscoelasticity, uniaxial tensile tests were used to characterize 
the dynamic response of the tissue. Different frameworks helped quantifying the tissue viscosity, the most common (Mijailovich et al., 1994; Navajas et al., 1995; Romero et al., 2011; Pinart et al., 2011; Yuan et al., 1997) being quasilinear viscoelasticity proposed by Fung that decouples the elastic and viscous phenomena and allows accounting for elastic non-linearities (Fung, 1993). In these studies, harmonic distorsion, loss and storage moduli as well as elastance, resistance and hysteresivity of the tissue were computed with the help of a Fourier transform. These parameters allow the investigation of the influence of loading factors - like stress amplitude, strain amplitude, frequency - or pathologies - like fibrosis (Dolhnikoff et al., 1999), acute lung injury (Ingenito et al., 1994; Rocco et al., 2001) - on the material parameters. A review of the elastic and viscous characterization of lung tissue, in particular using tests on excised samples, is given in (Suki and Bates, 2011). Another framework is proposed by (Holzapfel and Gasser, 2001) to account for the three-dimensional non-linear viscoelasticity of fiber-reinforced composites: the hyperelastic potential associated with the elastic behavior of the tissue is modified by a viscous contribution. This framework is particularly suited for a direct use in nonlinear continuum mechanics based simulations, this is why this approach is chosen in the present paper.

The aim of this paper is to determine a non-linear viscoelastic constitutive employing novel experimental and identification methods. The paper is divided into five parts: the first part covers the description of the experimental methods, particularly the optical method to measure the displacement field on the sample. In the second part, the numerical model and the constitutive law proposed for the lung parenchyma are detailed. The third, fourth 
and fifth parts are focused on the identification method, the results and the discussion, respectively.

\section{Uniaxial tensile tests on living PCLS}

Since the preparation of the samples and the experimental set-up are described in a previous paper (Rausch et al., 2011), only the main features are recalled here.

\subsection{Preparation of the samples}

The Precision-Cut Lung Slices (PCLS) are prepared from three isolated rat lungs as previously described in (Martin et al., 1996). The lungs are dissected from the animal, filled with an agarose solution (1.5\%) via the trachea and put on ice to allow the agarose to cool down and solidify. The lung lobes are separated and cut into tissue cylinders using a coring tool. The obtained cylinders have a diameter of $14 \mathrm{~mm}$; they are then cut into slices using a Krumdieck tissue slicer (Alabama Research and Development, Munford, AL). The thickness is set to $400 \mu \mathrm{m}$. The two sides of the round slices are trimmed with two parallel razor blades giving the tissue strip a width of $7.0 \mathrm{~mm}$. The strips are incubated in minimal essential medium (MEM) and the agarose is washed out by frequently changing the medium within the first 4 hours and completing an overnight incubation. As shown in (Martin et al., 1996), the obtained PCLS are viable for three days. In this protocol, the rat samples are tested within 48 hours after death, which guarantees that the tissue is still living while being tested (Martin et al., 1996) ; preliminary studies showed that the mechanical properties are not changing within 3 days (data not shown). The samples are kept in saline 
solution in the fridge $\left(4^{\circ} C\right)$ until they are tested. Tab. 1 sums up the specimen numbers.

\begin{tabular}{cccc}
\hline Name & \multicolumn{3}{c}{ Number of samples per frequency } \\
\hline & $0.2 \mathrm{~Hz}$ & $0.4 \mathrm{~Hz}$ & $0.8 \mathrm{~Hz}$ \\
\hline $\mathrm{G}$ & 7 & 5 & 4 \\
$\mathrm{I}$ & 4 & 4 & 4 \\
$\mathrm{~J}$ & 3 & 3 & 4 \\
\hline
\end{tabular}

Table 1: Samples description.

\subsection{Experimental set up}

The samples are tested using a Bose ElectroForce 3100 uniaxial device (Bose Cooperation, Eden Prairie, USA). The samples are fixed onto the machine using specifically designed clamps to prevent sliding and damage (Fig. 1). The moving clamp displacement is recorded using the internal displacement sensor of the tensile machine (range of $\pm 2.5 \mathrm{~mm}$ with a resolution of $12.5 \mu \mathrm{m})$; the other clamp is connected to the machine through a force sensor (range of $\pm 0.5 \mathrm{~N}$ with a resolution of $2.5 \mathrm{mN}$ ). Finally, a digital microscope is placed perpendicular to the sample plane to record the test. The images provided by the microscope are $640 \times 480 \mathrm{px}^{2}$.

Images of each test are recorded to perform image registration. Such an optical method allows the computation of the displacement field at the surface of the sample. It consists in mapping images of the sample in its undeformed and deformed state. This mapping is based on the gray-level distribution of the image; it requires for the images a good resolution and a stable gray-level distribution which has to exhibit a random pattern. If 


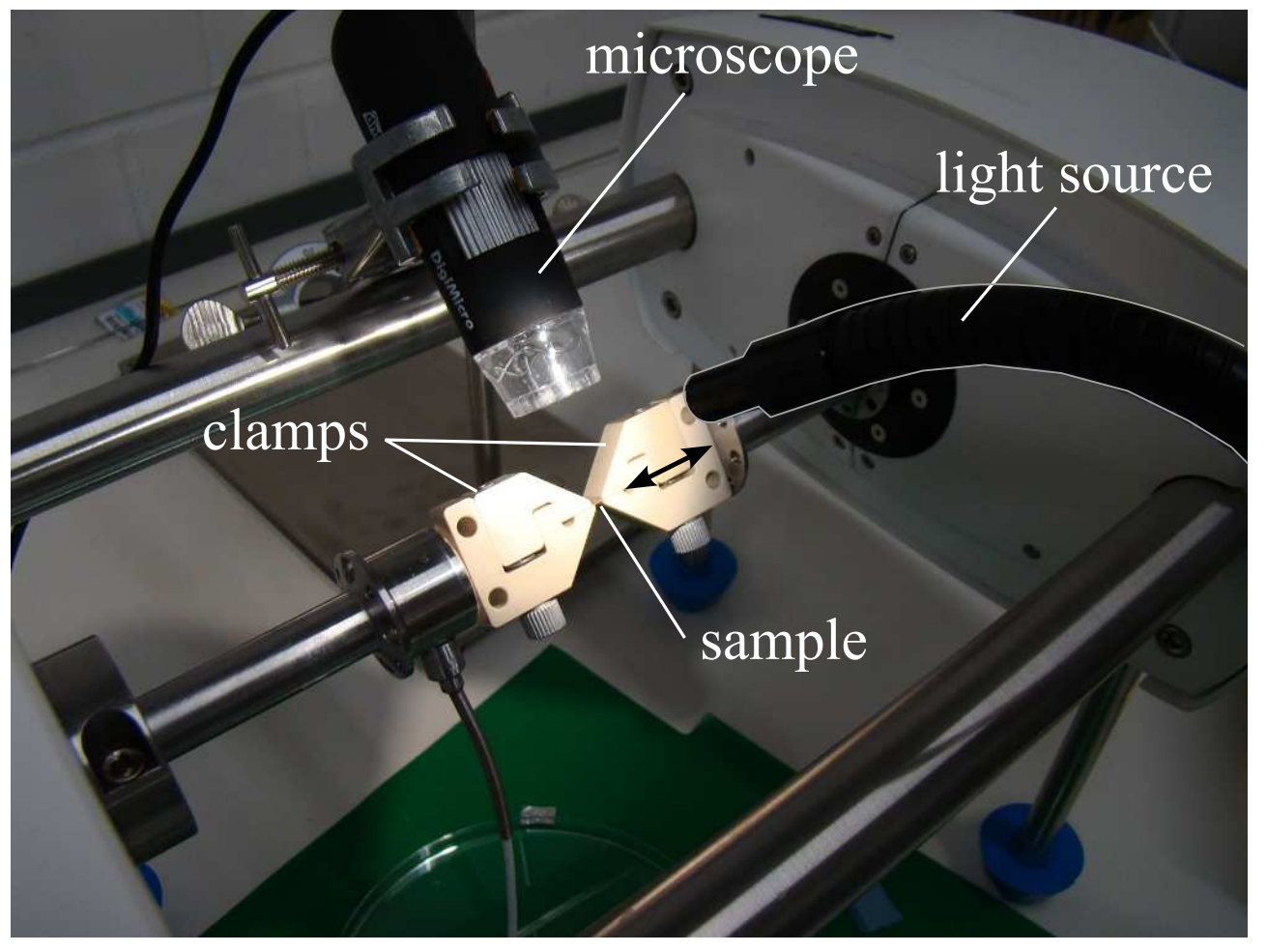

Figure 1: Experimental setup. The double arrow shows the tensile direction. 
the gray-levels are affected by a modification of the lightening or by light reflections at the surface of the sample, artifacts will occur in the computation of the displacement field.

In our case, the registration procedure relies on the microstructure of the lung parenchyma. By using a lightening that goes partially through the sample, this microstructure can be visualized; hence, the application of an additional random pattern using paint - as it is usually necessary when using an optical method to compute the experimental displacement - is superfluous. However, due to the low density of the tissue, the light rays intensity is hardly lessened when going through the tissue. Putting the light source and the microscope directly opposite to each other on both sides of the sample leads to a general overexposure, as well as a modification in the overall light intensity in the image during the sample stretching (since the sample gets thinner while being stretched, the penetration of light gets stronger). The solution to have a good insight of the microstructure and a satisfactory light intensity for the images was to put the light source on one side and slightly above the sample (Fig. 1); therefore the light is slightly scattered which reduces reflecting signals. The drawback of this method is that it does not completely prevent the occurrence of shining points, where the light is reflected on the wet surface of the sample. Even if not numerous, these parasite pixels can create artifacts during the registration procedure. However, the image registration algorithm helps smoothing these possible artifacts. 


\subsection{Testing protocol}

\subsubsection{Thickness measurement and placement on the machine}

The first step of the experimental protocol is to measure the actual thickness of the sample. Although the cutting tool is very accurate, the softness of the lung tissue and its compressibility inevitably lead to a variability in the thickness. To accurately measure the global sample thickness, a light microscope and a micrometer are used as shown in Fig. 2. Two microscopic slides are placed on each other and under the microscope; a pattern is drawn on the upper one. The microscope is focused on the pattern and the vertical position of the microscope plate is measured by a micrometer. The sample is then placed between the two microscopic slides and the water is wiped up so as not to create a water film between the two microscopic slides. At this stage the upper slide is in a higher position due to the thickness of the sample and the microscope does not focus on the pattern anymore. Then, the focus is adjusted on the upper slide pattern again; the resulting displacement of the microscope plate is the thickness of the sample, measured by the micrometer. The thickness is measured within an accuracy of $10 \mu \mathrm{m}$.

Once the thickness has been measured, the sample is hydrated again with saline solution. It is placed on a piece of aluminum foil. The foil and sample are placed in the clamps which are slightly fastened until the sample is sufficiently fixed to be able to remove the aluminum. The two clamps are then more firmly fastened to prevent sliding but also possible damage of the tissue. Finally, the extra water content is mopped up with paper so as to avoid measuring artificial forces due to surface tension effects of the water layer. 

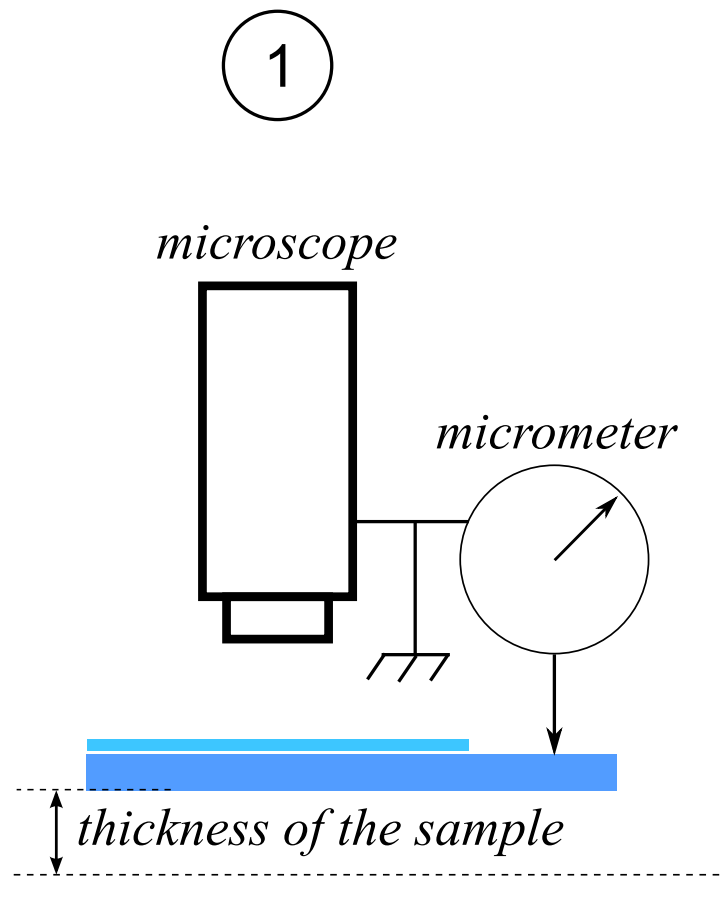

(2)

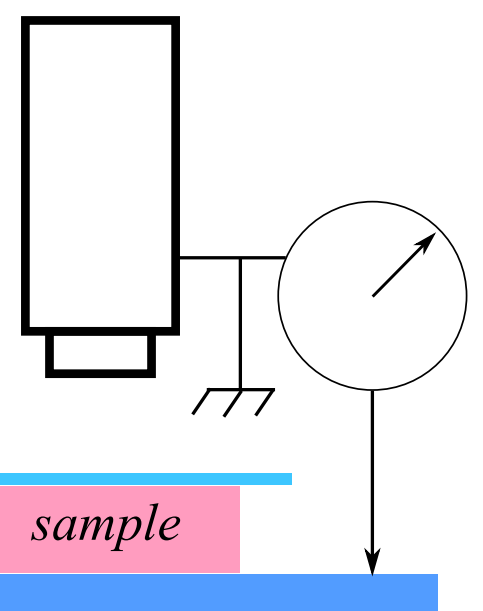

Figure 2: Method to measure the thickness of the sample: first, we focus the microscope on the upper slide without sample. Second, once the sample is put between the two slides, the microscope is focused again on the upper slide and the displacement of the microscope plate is measured by a micrometer. The measured displacement is the thickness of the sample. 
The initial length of the sample, i.e. the initial distance between the clamps, is set to $2.0 \mathrm{~mm}$.

\subsubsection{Preconditioning and tensile test}

In this work, the aim is to investigate the tissue behavior in the range of large strains. Preliminary tensile tests conducted on lung samples showed that when stretched to $50 \%$ strain and released, the sample exhibits a residual strain. Besides, during cycling tensile loading, the viscoelastic nature of the tissue leads to a transitory phase where the force-displacement cycles cannot be superimposed. One can assume that during breathing, the tissue exhibits a rather repeatable behavior from one cycle to the other. Therefore, a preconditioning phase is applied to the tissue to first, stabilize its residual strain and second, to stabilize the hysteresis loop obtained while cycling the sample.

In the present case, the preconditioning includes two main steps: first, the sample is stretched from 0 to $0.2 \mathrm{~mm}$ at a constant velocity of $0.5 \mathrm{~mm} / \mathrm{s}$ and maintained $10 \mathrm{~s}$ at $0.2 \mathrm{~mm}$; then this phase is repeated for $0.4,0.6$ and $0.8 \mathrm{~mm}$. The tissue is then released to a stretch of $0.2 \mathrm{~mm}$ for $30 \mathrm{~s}$. Second, the tissue is cycled between 0.2 and $1.2 \mathrm{~mm}$ for 10 cycles. The nine first cycles are used for preconditioning while the tenth is the actual tensile test. We found that nine cycles were enough to stabilize the cycle (see Sec.5.1).

\subsubsection{Reference state}

The preconditioning is performed to stabilize the residual strain; it leads to the definition of the stress-free reference state which is different from the initial state. We choose to perform the tensile test in a range that makes 
the sample slightly buckle at the minimum stretch. Therefore, when cycling, the force is slightly negative (compressive force) and becomes positive. The state corresponding to a force equal to zero is defined as the reference state for the image registration procedure. In practice, the zero-force state does not necessarily match one of the registration images for sampling frequency reasons; in that case, we choose the image that corresponds to the smallest force to define the reference geometry of the sample. Over all the samples, the initial force is always below $5 \%$ of the maximal force of the test. We also assume that the difference between reference and initial configurations is small enough to consider that the initial thickness is still applicable to the reference configuration. Indeed, the reference state corresponds experimentally to a clamp displacement of around $0.1 \mathrm{~mm}$.

\subsection{Postprocessing by image registration}

Using a full-field measurement method when testing soft material has several advantages. First, cutting samples of soft tissues of accurate dimensions is a difficult task; even with precision-slicing apparatus and procedure as described in Section 2.1, one cannot always obtain perfectly rectangular samples. Then, such thin samples are difficult to handle and to place in the clamps. The method described previously eases this procedure but we can for instance not perfectly control the orientation of the sample with respect to the loading direction. Finally, screwing the clamps to properly prevent sliding without damaging the sample requires some care; it can happen that the sample is not squeezed enough and experiences sliding during the test. For these reasons, it makes sense to use optical measurements additional to the force and global displacement information to know more accurately the 
actual displacement of the sample during the experiment.

Besides, the present work is focused on a compressible tissue. The identification procedure therefore requires information about the evolution of the width of the sample, which is directly related to the compressibility of the tissue. Global longitudinal measurement would not be sufficient to accurately identify the compressibility material constant.

As mentioned before, image registration consists in mapping two images of the sample that represent two different deformation states. These two images are called source image and target image; they both contain a scalar field that is a gray-level distribution over the pixels of the image. Image registration consists in finding the displacement field that minimizes the gap between the target image and the source image once it is deformed by this displacement field. We describe here the main features of this minimization procedure; more detailed information can be found in the cited papers.

The source and target images are denoted by $I_{s}(\mathbf{X})$ and $I_{t}(\mathbf{x})$ respectively where $\mathbf{x}$ and $\mathbf{X}$ are the spatial and material coordinates respectively.

For a so-called forward transformation, registering the two images consists in minimizing the error $\mathcal{D}$ with respect to the transformation $\varphi$ (Haber et al., 2009):

$$
\mathcal{D}[\varphi(\mathbf{X})]=\int_{\Omega_{x}}\left[I_{s}\left(\varphi^{-1}(\mathbf{x})\right)-I_{t}(\mathbf{x})\right]^{2} d x
$$

256 where $\varphi: I_{s} \mapsto I_{t}$ (Fig. 3) so that $\mathbf{x}=\varphi(\mathbf{X})=\mathbf{X}+\mathbf{u}$ with $\mathbf{u}$ a spatially varying displacement field. The solution $\mathbf{u}^{*}$ of the minimization is written: 


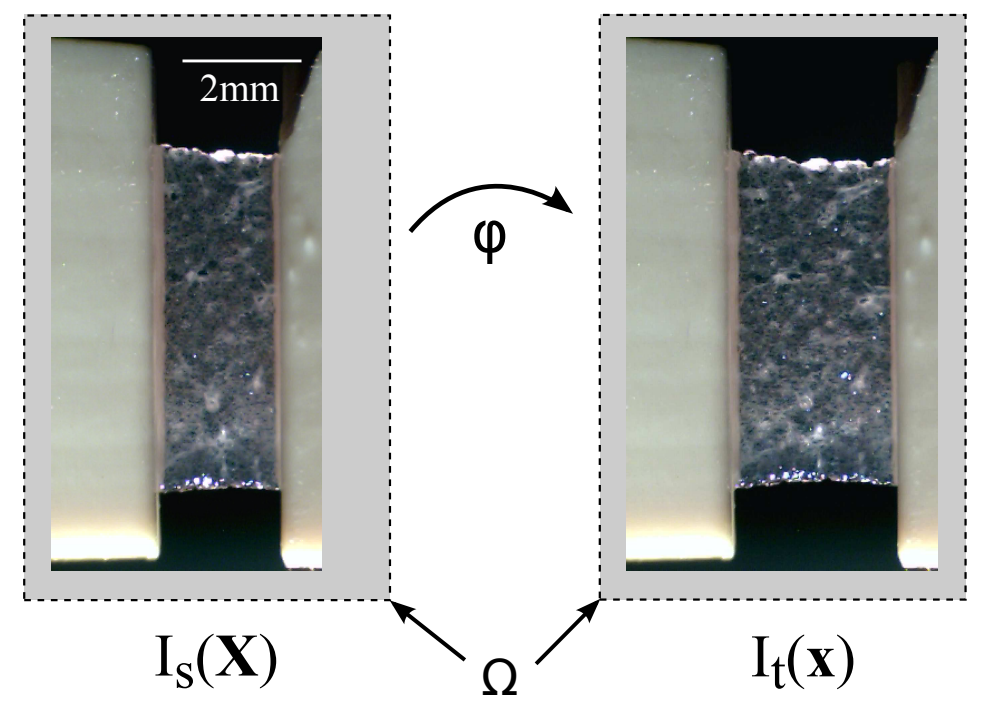

Figure 3: Source image (left) and target image (right).

$$
\mathbf{u}^{*}=\underset{\mathbf{u} \in L^{2}}{\arg \min } \mathcal{D}
$$

An elastic regularization $\mathcal{R}$ is added to ensure the well-posedness of the optimization problem (Modersitzki, 2004; Fischer and Modersitzki, 2008).

The minimization is therefore:

$$
\mathbf{u}^{*}=\underset{\mathbf{u} \in L^{2}}{\arg \min }\left[\mathcal{D}\left[I_{s}, I_{t}\right]+\alpha \mathcal{R}(\mathbf{u})\right]
$$

where the weighting factor $\alpha$ is set to 0.1 in this work.

We minimize $\mathcal{D}$ using a Gauss-Newton optimization scheme (Haber and Modersitzki, 2006) as well as a multiresolution approach (Lester and Arridge, 1999) (Fig. 4). First, from the pixel-size grid, i.e. the image recorded during the experiment, several coarser and coarser grids are successively created by filtering and sampling the gray-level field of the image at the one-level finer 
scale. We obtain $n$ levels of resolution. The minimization starts at the coarser level $n$; the result $\mathbf{u}_{n}$ of the minimization of $\mathcal{D}$ is linearly interpolated over the grid at level $n-1 ; \mathbf{u}_{n}^{n-1}$ is used as the initial guess for the minimization at level $n-1$. This procedure is repeated until $\mathbf{u}_{1}=\mathbf{u}^{*}-$ at the pixel level is determined.

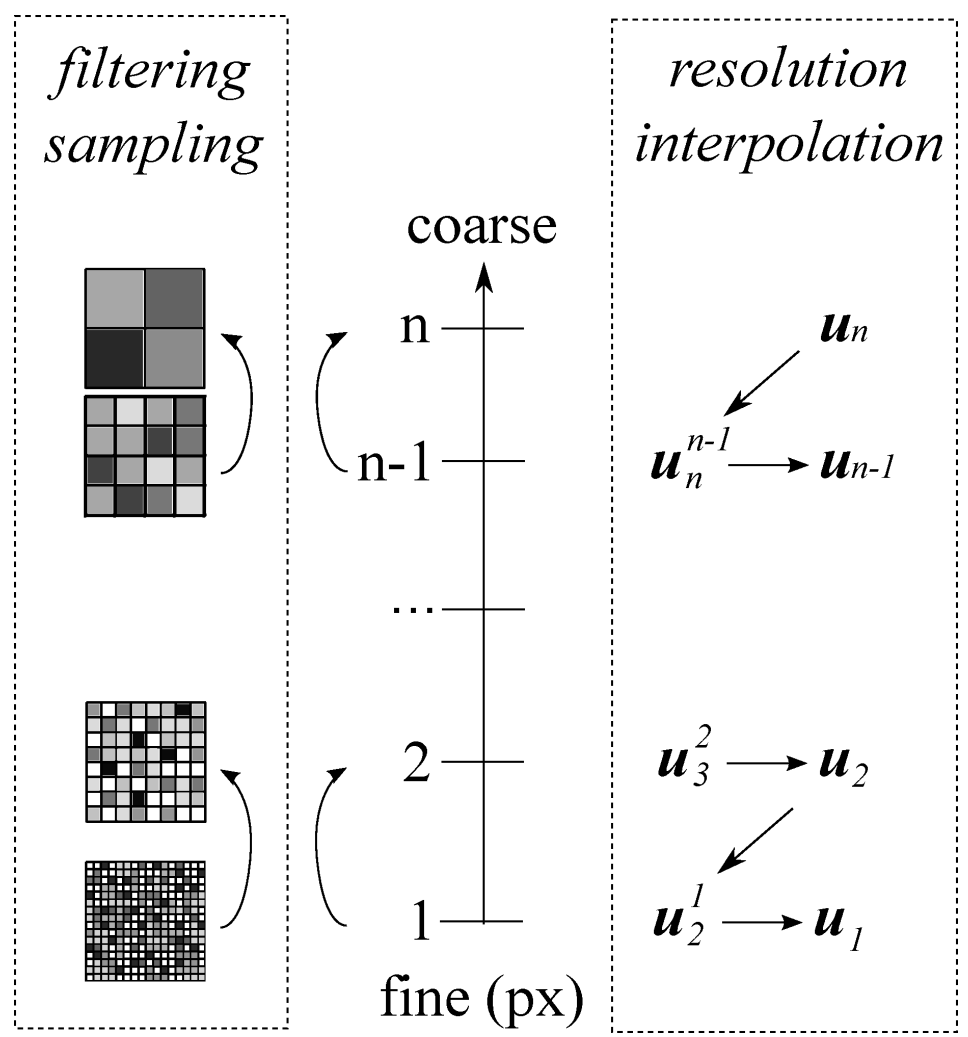

Figure 4: Multiresolution approach to solve the minimization problem of the image registration. $\mathbf{u}_{i}$ is the solution of the minimization at level $i . \mathbf{u}_{i}^{j}$ is the linear interpolation of $\mathbf{u}_{i}$ over level $j$ and is the initial guess for the resolution at level $j . \mathbf{u}_{1}=\mathbf{u}^{*}$ is the solution of the problem. The finer grid (lower left) is the image recorded during the test.

In our case, five levels of resolution are used. The image registration algorithm is implemented in Matlab, as well as the post-processing of the 
registration results (creation of the input file for the simulation).

The overall registration procedure can be computed in two different ways: sequentially or incrementally. The sequential method consist in defining a reference image (corresponding to the reference state of the sample) and register all the other images (while the sample is stretched) with respect to this reference image. The incremental method consists in updating the reference image: image $n+1$ is registered with respect to image $n$. In this case, the Finite Element mesh (see next section) needs to be updated at each new reference image: the position of the nodes of the FE-mesh at step $n$ is updated using the result of the registration between images $n-1$ and $n$. Sec. 5.2 gives a comparison of the two methods.

The images provided by the microscope are $640 \times 480 \mathrm{px}^{2}$, corresponding to $15.2 \times 11.4 \mathrm{~mm}^{2}$. The registration procedure guarantees an accuracy of a pixel size of $0.023 \mathrm{~mm}$.

\section{Numerical model of the lung parenchyma samples}

\subsection{Finite Element model}

The numerical identification procedure is based on the simulation of the experimental test and the minimization of the error between the simulated and the experimental displacements. In order to simulate the test, a zone of interest (ZOI) is defined on the reference image and a Finite Element mesh is created on this ZOI. The shape of the ZOI is quadrangular but not necessarily rectangular. The registration procedure is computed on the entire image but only the results in the ZOI are further used for the identification. The Matlab function interp 2 is used to interpolate the computed displacements on the 
nodes of the FE-mesh. The thickness that was experimentally measured is defined as the initial thickness of the simulated sample.

The simulation is performed using our in-house multi-purpose research software platform $B A C I$. The input data for the simulation of the test are the FE-mesh, the material law with the initial guess of parameters and the boundary conditions.

\subsection{Constitutive law}

The aim of this study is to identify a suitable visco-hyperelastic constitutive model for lung parenchyma and determine the corresponding model parameters. The model that we use in the present case is a generalized Maxwell model with different hyperelastic contributions.

The hyperelastic contribution is described in details in (Rausch et al., 2011). Briefly, a hyperelastic toolbox is implemented in $B A C I$ which allows the combination of several different Strain Energy Functions (SEF). We only mention here the isotropic SEF as the lung parenchyma is isotropic. The SEF are of two types: coupled and decoupled. A coupled SEF is based on the overall strain tensor, while a decoupled SEF is split into volumetric and isochoric contributions. The decoupled contributions are all polyconvex and stress-free in the reference state, which allows to sum them up without further condition.

In our case, only decoupled potentials are used due to the higher variety of available potentials of this form than for coupled potentials.

The viscous contribution is based on the work of (Holzapfel and Gasser, 2001). A viscous part is added to the hyperelastic contribution based on a generalized Maxwell model. In this paper, we consider the basic form of the 


$$
\begin{gathered}
\dot{\mathbf{Q}}+\frac{1}{\tau} \mathbf{Q}=\beta \dot{\mathbf{S}} \\
\left.\mathbf{Q}\right|_{t=0}=\mathbf{0}
\end{gathered}
$$

$$
\psi=\psi^{\infty}\left(I_{i}\right)+\gamma(\mathbf{C}, \boldsymbol{\Gamma})
$$

where $\psi^{\infty}$ is the hyperelastic potential and $\gamma$ is the dissipative potential. This theoretical framework is detailed for the isotropic case in Appendix A; details for anisotropic cases can be found in (Holzapfel and Gasser, 2001).

Similar to the right Cauchy-Green strain tensor $\mathbf{C}$ being the kinematic conjugate of the elastic second Piola-Kirchhoff stress tensor $\mathbf{S}, \boldsymbol{\Gamma}$ is the kinematic conjugate of the fictitious stress tensor $\mathbf{Q}$. These stress tensors are expressed by:

$$
\begin{aligned}
\mathbf{S}^{\infty} & =2 \frac{\partial \psi^{\infty}}{\partial \mathbf{C}} \\
\mathbf{Q} & =-2 \frac{\partial \gamma}{\partial \boldsymbol{\Gamma}}
\end{aligned}
$$

$\mathbf{Q}$ is a non-equilibrium stress; therefore, $\mathbf{Q}=\mathbf{0}$ when $t \rightarrow \infty$. The evolution of $\mathbf{Q}$ is governed by an equation of evolution:

334 where $\tau$ defines a relaxation time and $\beta$ is a non-dimensional free-energy 335 factor. The global stress tensor is therefore $\mathbf{S}=\mathbf{S}^{\infty}+\mathbf{Q}$. The elasticity 
tensor is also expressed as the sum of an elastic summand and a viscous summand (Eq. 9)

$$
\mathbb{C}=\mathbb{C}^{\infty}+\mathbb{C}^{\text {vis }}
$$

Tab. 2. Only isochoric potentials involving the first invariant are identified as it was shown in (Rausch et al., 2011) that potentials with both the first and the second invariants did not lead to a significant decrease of the error in the identification. We compared several combinations of hyperelastic potentials to find out which one gives the best fit. Tab. 3 displays the tested combinations.

\section{Identification method}

The identification procedure consists in minimizing the error between computed and experimental displacements for the last cycle, i.e. both the loading and unloading curves are taken into account. The input data for this inverse analysis are the simulation data mentioned before and the experimental displacements at the nodes of the FE-mesh that are taken into account for the cost function calculation - typically all the degrees of freedom of the FE-mesh nodes that are not kinematically constrained (Fig. 5). 


\begin{tabular}{|c|c|c|}
\hline Vol. & Sussman-Bathe & $\psi_{v s b}=\frac{\kappa}{2}(J-1)^{2}$ \\
& Ogden & $\psi_{v o}=\frac{\kappa}{4}\left(-2 \ln J+J^{2}-1\right)$ \\
& Penalty & $\psi_{v p}=\epsilon\left(J^{\gamma}+\frac{1}{J^{\gamma}}-2\right)$ \\
\hline Iso. & Polynomial order n & $\psi_{i p n}=c_{n}\left(\overline{I_{1}}-3\right)^{n}$ \\
& Exponential & $\psi_{i e}=\frac{k_{1}}{2 k_{2}}\left[e^{k_{2}\left(\frac{\bar{I}}{3}-1\right)}-1\right]$ \\
\hline
\end{tabular}

Table 2: Volumetric and isochoric hyperelastic potentials tested in this work. Parameters in red are to be identified. Sussman-Bathe from (Doll and Schweizerhof, 2000). Ogden from (Ogden, 1974). Penalty from (Balzani et al., 2006). Polynomial from a split and extension of Yeoh's potential (Yeoh, 1993). Exponential from (Delfino et al., 1997).

\begin{tabular}{|c|c|}
\hline $\mathrm{A}$ & $\psi=\psi_{v o}+\psi_{i p 1}+\psi_{i p 2}+\psi_{i p 3}$ \\
$\mathrm{~B}$ & $\psi=\psi_{v o}+\psi_{i p 1}+\psi_{i p 3}$ \\
$\mathrm{C}$ & $\psi=\psi_{v o}+\psi_{i p 1}+\psi_{i p 2}$ \\
$\mathrm{~K}$ & $\psi=\psi_{v o}+\psi_{i p 1}+\psi_{i p 4}$ \\
$\mathrm{P}$ & $\psi=\psi_{v o}+\psi_{i e}$ \\
\hline
\end{tabular}

Table 3: Identified hyperelastic potentials. 


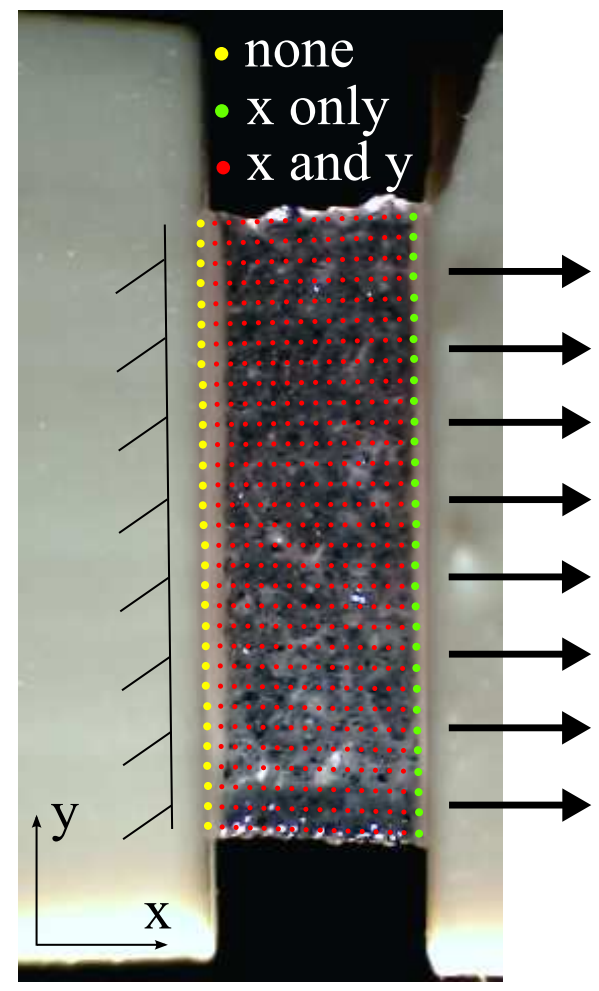

Figure 5: Degrees of freedom used for the inverse analysis. The nodes at the fixed clamp are not used. Only the x-displacement of the moving clamp nodes is used; for the other nodes, the two degrees of freedom are used. 
The minimization follows a pseudo-transient continuation type scheme. The constitutive parameters of each summand of the material law and the associated viscous contribution are stored in the parameter vector $\mathbf{p}$; its size is $n_{p}$. For each loop of the inverse analysis, $n_{p}+1$ simulations are performed: $n_{p}$ simulations with only one modified element of $\mathbf{p}(p(i)=p(i)+$ $a+b \times p(i)$, where $a$ and $b$ are user-defined perturbation parameters) and one simulation with unperturbed $\mathbf{p}$. These simulations allow the determination of the Jacobian matrix $\mathbf{J}$ of the problem (Eq. 10) and the computation of the new set of parameters $\mathbf{p}=\mathbf{p}+\Delta \mathbf{p}($ Eq. 11,12)

$$
\begin{gathered}
\mathbf{J}=\left(\begin{array}{ccc}
\frac{\partial \mathbf{u}_{1}}{\partial p_{1}} & \cdots & \frac{\partial \mathbf{u}_{1}}{\partial p_{n_{p}}} \\
\vdots & & \vdots \\
\frac{\partial \mathbf{u}_{n}}{\partial p_{1}} & \cdots & \frac{\partial \mathbf{u}_{n}}{\partial p_{n_{p}}}
\end{array}\right) \\
\Delta \mathbf{p}=\left[\mathbf{J}^{T} \mathbf{J}+\mu \operatorname{diag}\left(\mathbf{J}^{T} \mathbf{J}\right)\right]^{-1} \mathbf{J}^{T} \mathbf{R} \\
\mathbf{R}=\left(\begin{array}{c}
\mathbf{r}_{1} \\
\vdots \\
\mathbf{r}_{n}
\end{array}\right)=\left(\begin{array}{c}
\mathbf{u}_{1, \text { sim }}-\mathbf{u}_{1, \exp } \\
\vdots \\
\mathbf{u}_{n, \text { sim }}-\mathbf{u}_{n, \exp }
\end{array}\right)
\end{gathered}
$$

where $\mathbf{u}_{i}$ is the displacement at node $i$. The criterion of convergence of the minimization is based on the gradient of the error $\nabla(e)$ where $e$ is the cost function, i.e. the sum of the error at all nodes and all time steps.

The parameters of the inverse analysis are therefore the convergence criterion, the maximum number of optimization loops, the perturbation parameters $a$ and $b$ and an initial regularization factor $\mu$. 
A nested parallelization implemented in $B A C I$ allows computing these simulations in parallel, each simulation being itself also computed in parallel; regardless of $n_{p}$, one loop of the inverse analysis lasts as long as the longest of the $n_{p}+1$ simulations, instead of $n_{p}+1$ times the time of one simulation. For each combination of one volumetric potential and possibly several isochoric potentials of Tab. 2, a purely hyperelastic identification is done first, then the viscous contribution is added, which means that an additional parameter $\tau$ is to be identified - in this work we set $\beta$ to 1 and do not identify it. The results of the first identification are used as initial guesses for the viscoelastic identification. During this second phase, both the hyperelastic and the viscous parameters are optimized.

\section{Results}

The identification results are statistically analyzed using the non-parametric Mann-Whitney test (Mann and Whitney, 1947); the p-factor is indicated when a tendency is significant.

\subsection{Force versus displacement curves}

Fig. 6 shows the evolution of the hysteresis loop during the ten cycles of the tensile loading. The hysteresis loop decreases and stabilizes; only the last cycle is taken into account for the identification.

\subsection{Comparison between sequential and incremental image registration}

The displacements obtained with the incremental and sequential methods are compared in Fig. 7 and 8. Fig. 7 shows that when the stretch increases (from image 4 to image 14 in that case), the gap between the two methods 


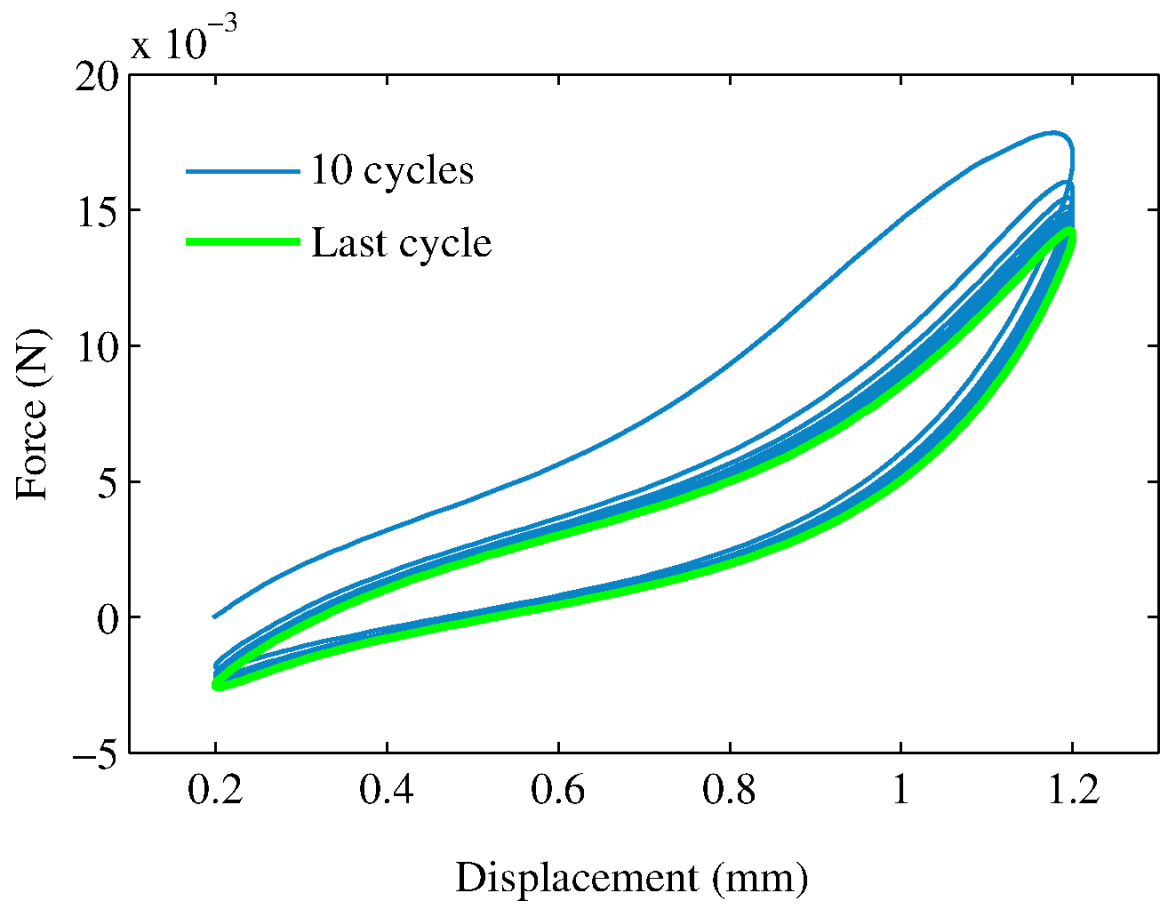

Figure 6: Example of measured curve over ten cycles of tension loading (sample from rat G, frequency: $0.4 \mathrm{~Hz}$ ). The displacement displayed here is measured by the sensor of the tensile machine. 
increases but remains small (less than $2 \%$ of the maximum displacement in $\mathrm{x}$-direction). Fig. 8 shows that the incremental method gives a slightly better result as the new position of the FE-mesh nodes corresponds better to the visually expected one (the displacement of the moving clamp nodes is better). That is why we choose the incremental method.

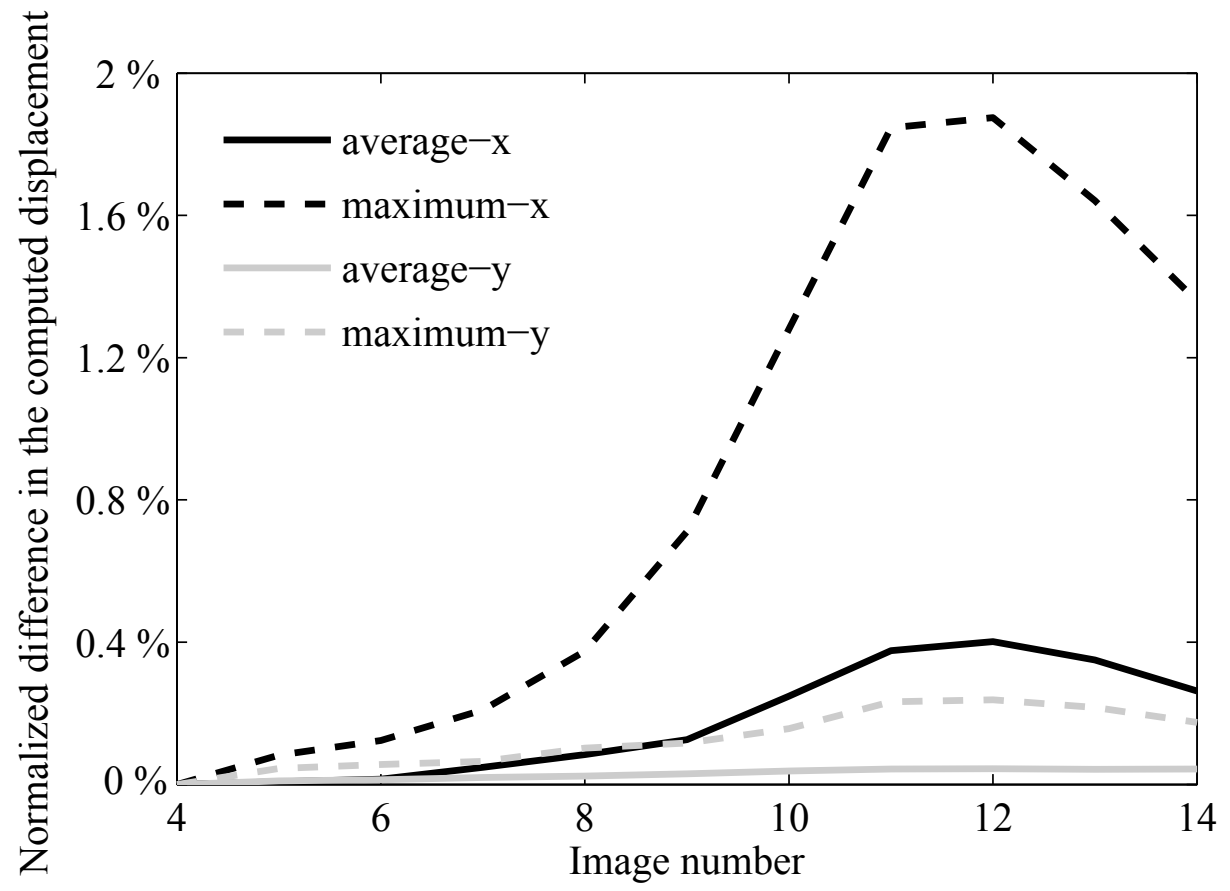

Figure 7: Difference of the displacements computed with the incremental and sequential image registration. At each image, average values and maximum values (over all the FEmesh nodes) of the gap in the $\mathrm{x}$ - and $\mathrm{y}$-directions are normalized by the maximum value of the $\mathrm{x}$ - and $\mathrm{y}$-displacements respectively. 


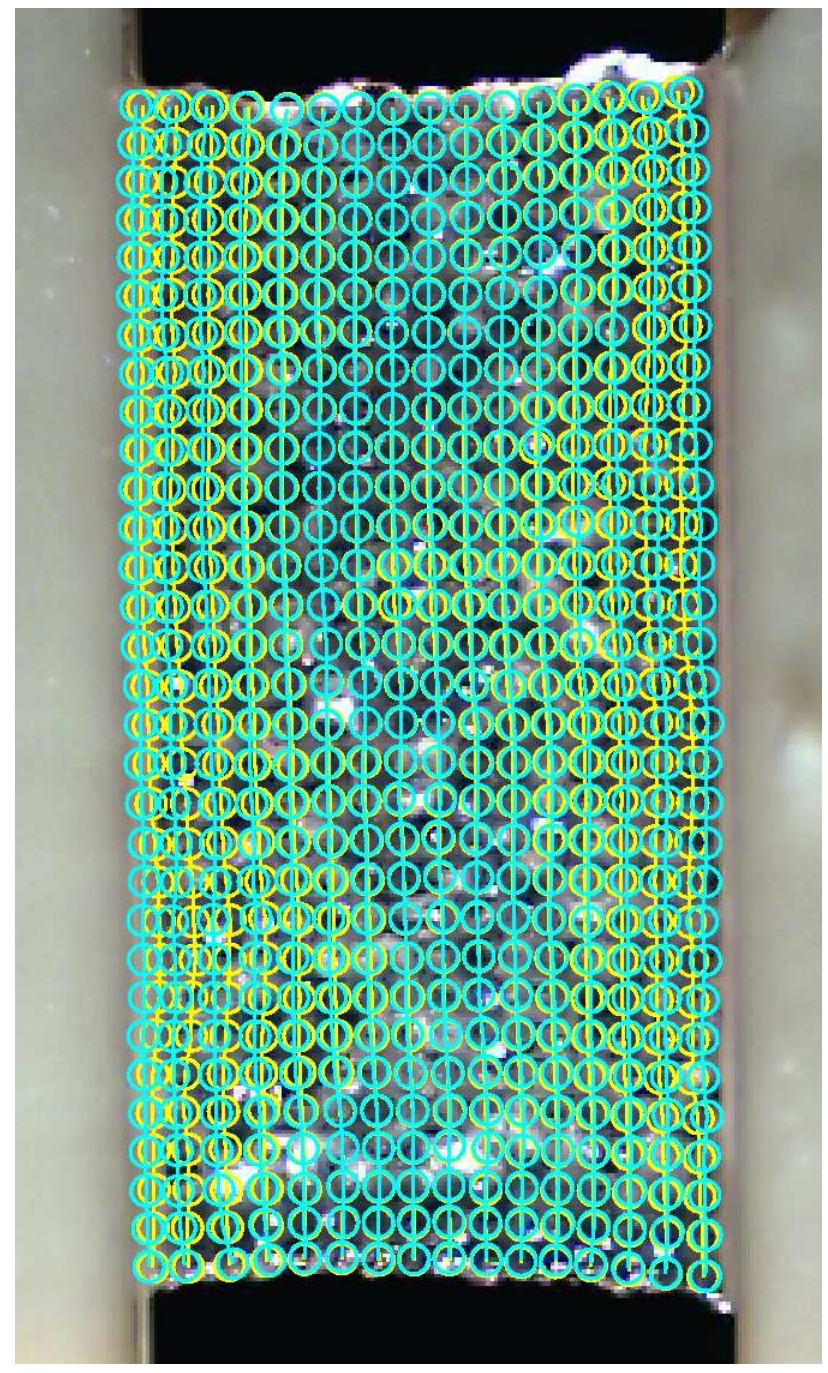

Figure 8: Position of the FE-mesh nodes superimposed on the test picture: position computed by the incremental (blue) and sequential (yellow) image registration methods, for a deformed image. The moving clamp is the right one. 


\subsection{Convergence of the identification procedure}

Parametric studies have been conducted to ensure that the present identification method is robust and valid. In the following, the criterion of convergence is $\nabla(e) \leq 10^{-6}$.

Convergence using artificial data. For this study, artificial data are generated using one of the experiments. A test is simulated with experimental load conditions and an arbitrary set $\hat{\mathbf{p}}$ of material parameters. The computed displacements are used to create a new input file. We then proceed to identify the material parameters of the same law using this file from an arbitrary initial guess, and check that the identified parameters coincide with $\hat{\mathbf{p}}$. This procedure has been conducted using a hyperelastic potential of the following form :

$$
\psi=\psi_{v o}+\psi_{i p 1}+\psi_{i p 3}
$$

For a total number of elements from 250 to 4410, the maximal value of the average error on the three identified parameters is $0.00731 \%$ (250 elements) which shows convergence to the right values (target values: $\kappa=30 \mathrm{kPa}, \mathrm{C}_{1}=$ $5 \mathrm{kPa}, \mathrm{C}_{3}=10 \mathrm{kPa}$; initial guess: $\left.\kappa=50 \mathrm{kPa}, \mathrm{C}_{1}=0.5 \mathrm{kPa}, \mathrm{C}_{3}=5 \mathrm{kPa}\right)$. These values were obtained after 9 to 11 identification loops.

To study the convergence of the viscoelastic identification, one viscous constant $\tau$ is added. In that case, four parameters are to be identified (target values: $\kappa=70 \mathrm{kPa}, \mathrm{C}_{1}=3.5 \mathrm{kPa}, \mathrm{C}_{3}=33 \mathrm{kPa}, \tau=0.1$; initial guesses: $\kappa=$ $\left.30 \mathrm{kPa}, \mathrm{C}_{1}=5 \mathrm{kPa}, \mathrm{C}_{3}=50 \mathrm{kPa}, \tau=0.05\right)$. A single FE-mesh size of 1210 elements is used. The identification leads to an error on the target value in 
the order of $1 \%$ for $\tau$ and $0.05 \%$ for the other parameters. The convergence is therefore very satisfactory.

Influence of the FE-mesh size. Fig. 9 shows the result of the identification of the law of Eq. (13) for different mesh sizes. All the identifications started from the same initial guess; they are conducted on a real test (not on generated data from known parameters as for the previous study). When the number of elements increases, the identified parameters converge to their optimized value, as the evolution of the error shows. The compromise between computational time and accuracy was set to around 1200 elements.

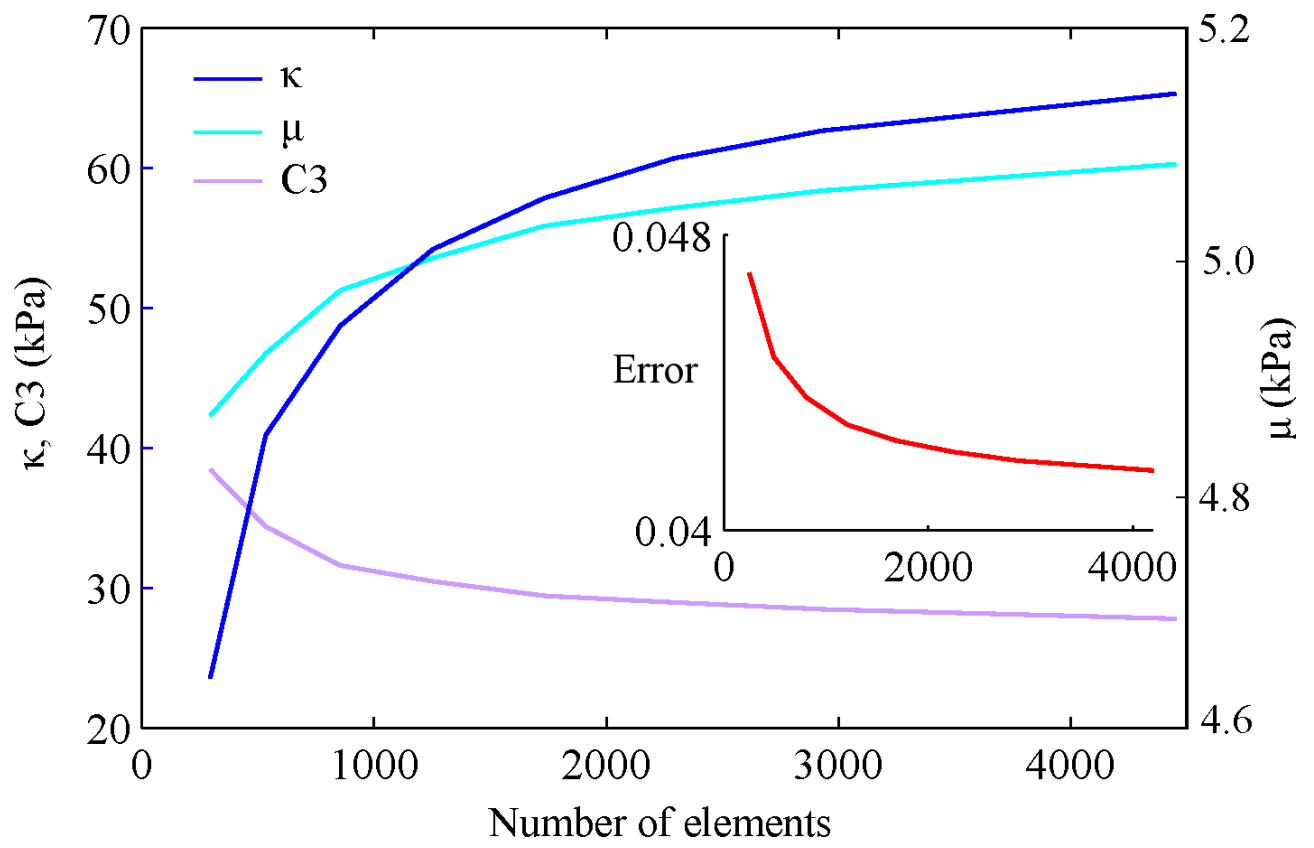

Figure 9: Evolution of the identified parameters for law $B$ (see Tab. 3) with respect to the total number of elements, for one sample of rat $\mathrm{G}$. The evolution of the error $e$ is displayed in red. 
Sensitivity analysis. We evaluated the sensitivity of the error to small variations of the constitutive parameters. Fig. 10 and 11 display the evolution of the error for the hyperelastic law given in Eq. (13) with a variation of $\pm 10 \%$ around the identified value of the parameters $\kappa, \mathrm{C}_{1}$ and $\mathrm{C}_{3}$, and for a viscoelastic law (potential of Eq. (13) + viscous contribution) with a variation of $\pm 20 \%$ around the identified value of the time parameter $\tau$. In both cases, when we look at the scales, the error map is rather flat, but it is however slightly influenced by small variations of the parameters. In particular, the first order parameter $\left(\mathrm{C}_{1}\right)$ has a strong influence, while $\mathrm{C}_{3}$ and $\kappa$ are rather coupled (the error displays a valley). However, we could check that a minimum exists by testing different initial guesses for all the samples and by checking that the identification always converges to the same set of parameters. For the viscoelastic identification, there is a clear minimum of the error with respect to $\tau$.

\subsection{Hyperelastic identification}

For both the hyperelastic and viscoelastic identifications, information on the thickness is required. The measured thickness for all the tested samples was $442 \pm 34 \mu \mathrm{m}, 503 \pm 57 \mu \mathrm{m}$ and $519 \pm 70 \mu \mathrm{m}$ for rats $\mathrm{G}, \mathrm{I}$ and $\mathrm{J}$ respectively. For different reasons (rupture, poor quality of the image registration) some of these tests could not be fully post-processed.

Volumetric potential. As mentioned in Sec.3.2, three volumetric potentials are tested. Fig. 12 displays the error obtained with these three volumetric potentials combined with the same isochoric potential of type $B$ (which parameters are not identified), for all the samples of a single rat. We can 

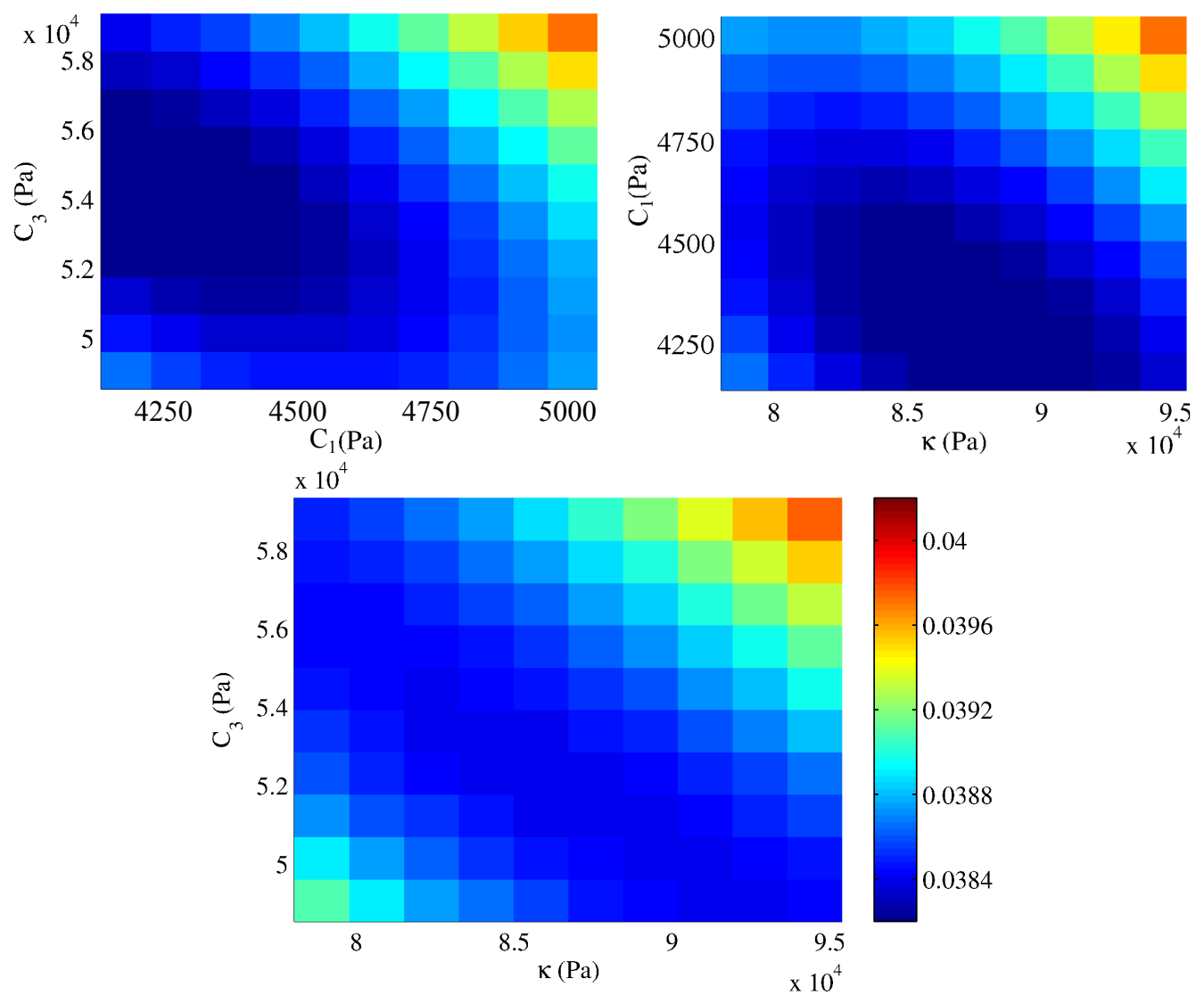

Figure 10: Sensitivity of the error to small variations of the constitutive parameters. The sensitivity study is conducted on a sample of rat I tested at $0.2 \mathrm{~Hz}$, the constitutive law is of type B. For each graph, the respective third parameter is kept constant. 


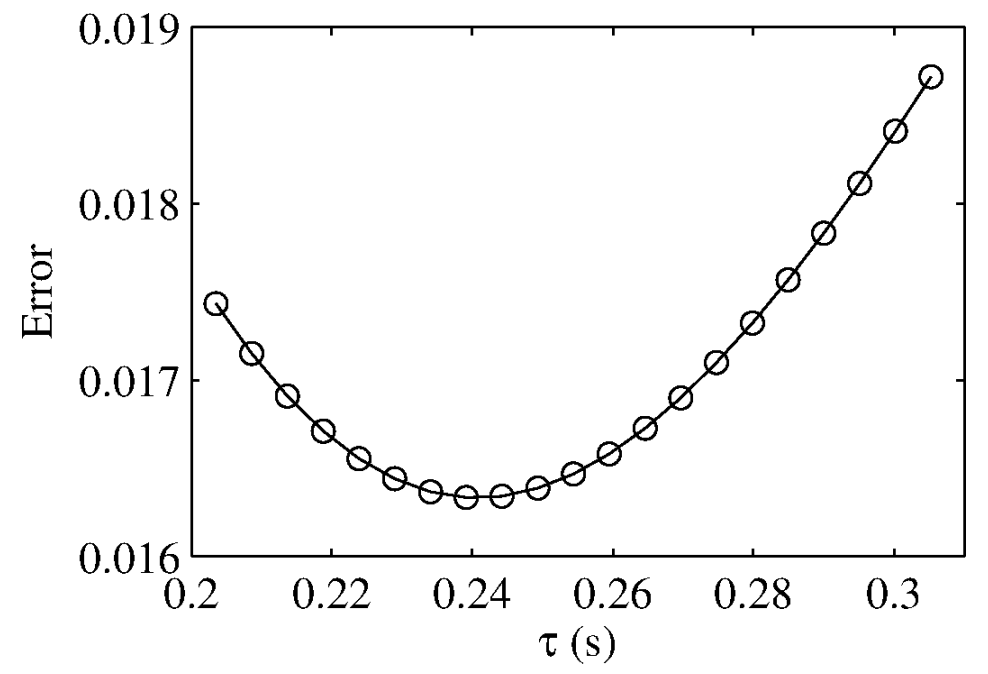

Figure 11: Sensitivity of the error to small variations of parameter $\tau$ of a viscoelastic law. The sensitivity study is conducted on a sample of rat I tested at $0.2 \mathrm{~Hz}$, the constitutive law is of type E. Parameters of the elastic contribution are kept constant.

see that Sussman-Bathes and Ogden potentials give equal results while the penalty potential is either equivalent or worse. Therefore, the volumetric potential used in the following parts will always be the Ogden form.

Identification of volumetric and isochoric parameters. Fig. 13 shows the results obtained for each tested frequency among all the tested samples (three rats). We can see the comparison between the combinations of potentials given in Tab. 3. This shows that the performances of these forms of potential give rather similar results, but the exponential form (law P) gives the best fit for each frequency. A Mann-Whitney statistical test computed on all the samples shows that law $\mathrm{C}$ gives significantly poorer results than laws A, B and $\mathrm{P}$.

Tab. 4 gives the value of the identified parameters for these hyperelastic 


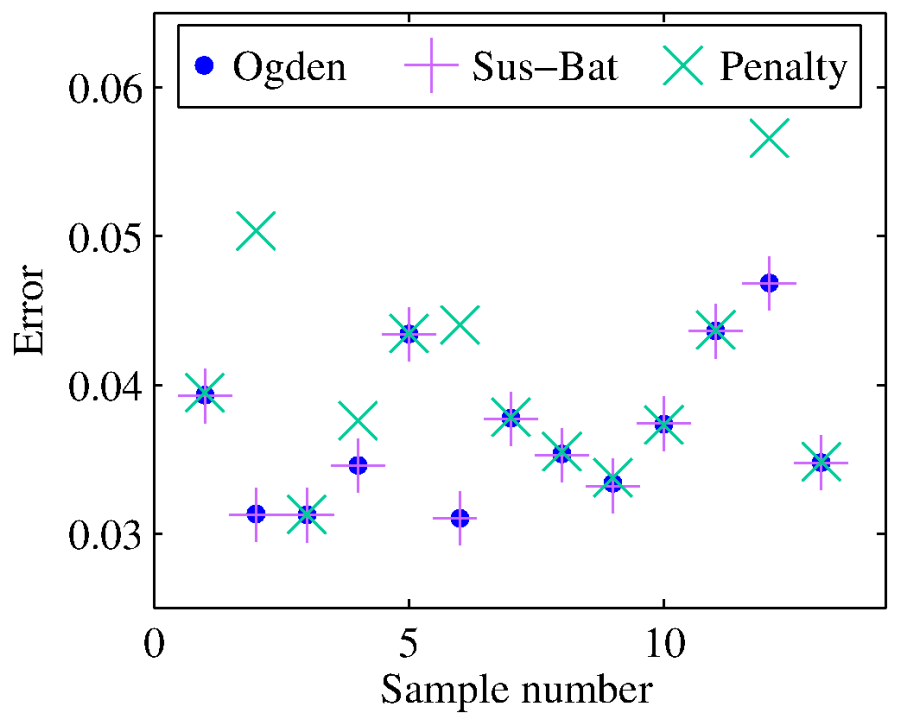

Figure 12: Comparison of the performance of Ogden, Sussman-Bathe and penalty volumetric potentials for parenchyma samples from the same rat. The volumetric potential is always associated to the same isochoric potential of type B (see Tab. 3).

potentials. As there is no statistical difference between the identified parameters for the varying tested frequencies, the values are averaged over all the samples and all the frequencies. The identified value of $\kappa$ is averaged over all the potentials as this value is independent from the form of the isochoric potential.

\subsection{Viscoelastic identification}

Fig. 14 displays an example of hyper- versus viscohyperelastic identification. For force versus displacement curves in the direction of the applied load, we can see that a hyperelastic potential represents the non-linearity of the material but only adding a viscous contribution can lead to the correct hysteresis. 


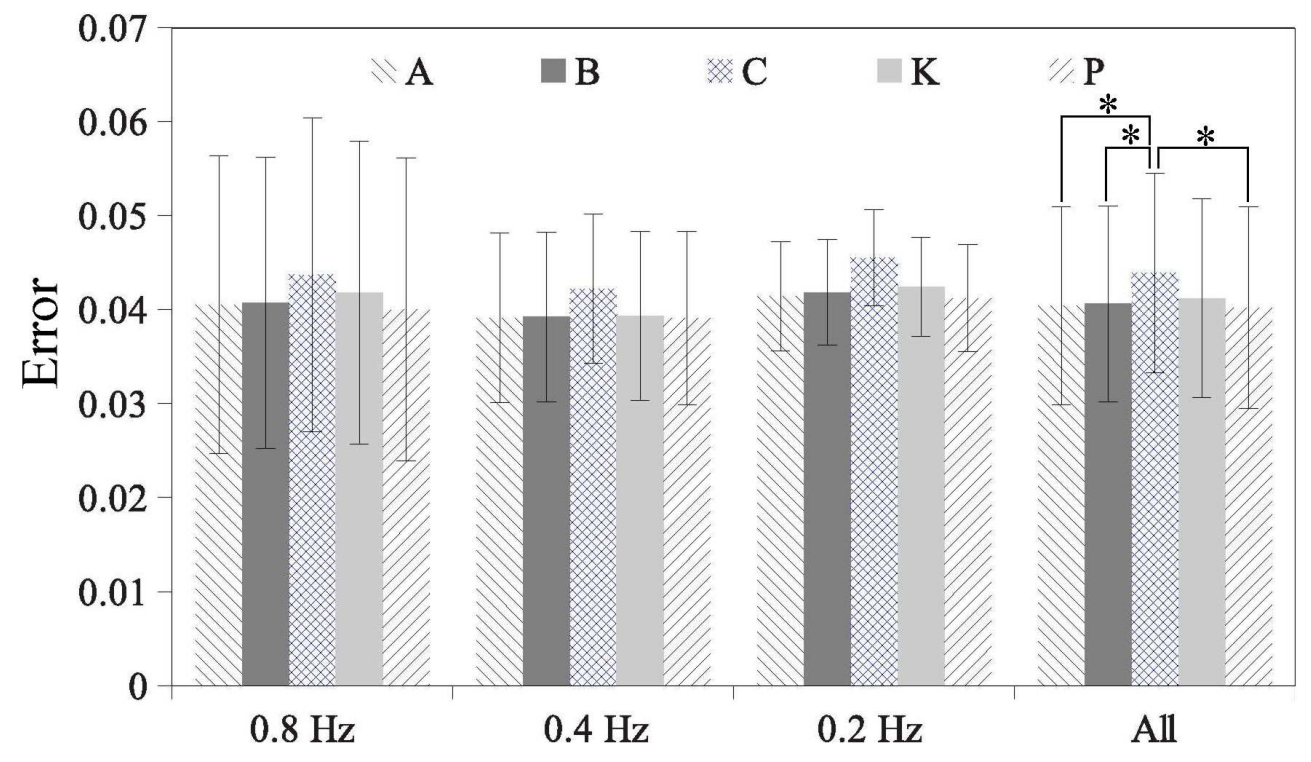

Figure 13: Comparison of the performance of different forms of hyperelastic potentials for various frequencies, among all the tested samples $(12$ at $0.8 \mathrm{~Hz}, 12$ at $0.4 \mathrm{~Hz}, 14$ at $0.2 \mathrm{~Hz}$, all from three different rats). The average $\pm \mathrm{SD}$ of the error is displayed. ${ }^{*}$ corresponds to $\mathrm{p} \leq 0.05$. 


\begin{tabular}{|c|c|c|c|}
\hline Law & Parameter & Value & Unit \\
\hline & $\kappa$ & $7.25 \mathrm{e} 4 \pm 2.74 \mathrm{e} 4$ & $\mathrm{~Pa}$ \\
\hline & $C_{1}$ & $2.45 \mathrm{e} 3 \pm 8.95 \mathrm{e} 2$ & $\mathrm{~Pa}$ \\
$\mathrm{~A}$ & $C_{2}$ & $3.69 \mathrm{e} 3 \pm 1.96 \mathrm{e} 3$ & $\mathrm{~Pa}$ \\
& $C_{3}$ & $3.22 \mathrm{e} 4 \pm 1.81 \mathrm{e} 4$ & $\mathrm{~Pa}$ \\
\hline $\mathrm{B}$ & $C_{1}$ & $2.87 \mathrm{e} 3 \pm 9.05 \mathrm{e} 2$ & $\mathrm{~Pa}$ \\
\hline $\mathrm{K}$ & $C_{3}$ & $3.83 \mathrm{e} 4 \pm 1.63 \mathrm{e} 4$ & $\mathrm{~Pa}$ \\
\hline $\mathrm{P}$ & $C_{1}$ & $3.43 \mathrm{e} 3 \pm 1.05 \mathrm{e} 3$ & $\mathrm{~Pa}$ \\
& $C_{4}$ & $1.34 \mathrm{e} 5 \pm 8.30 \mathrm{e} 4$ & $\mathrm{~Pa}$ \\
\hline & $k_{2}$ & $5.70 \mathrm{e} 3 \pm 1.60 \mathrm{e} 3$ & $\mathrm{~Pa}$ \\
\hline
\end{tabular}

Table 4: Identified parameters (average $\pm \mathrm{SD}$ ) for purely elastic potentials. 

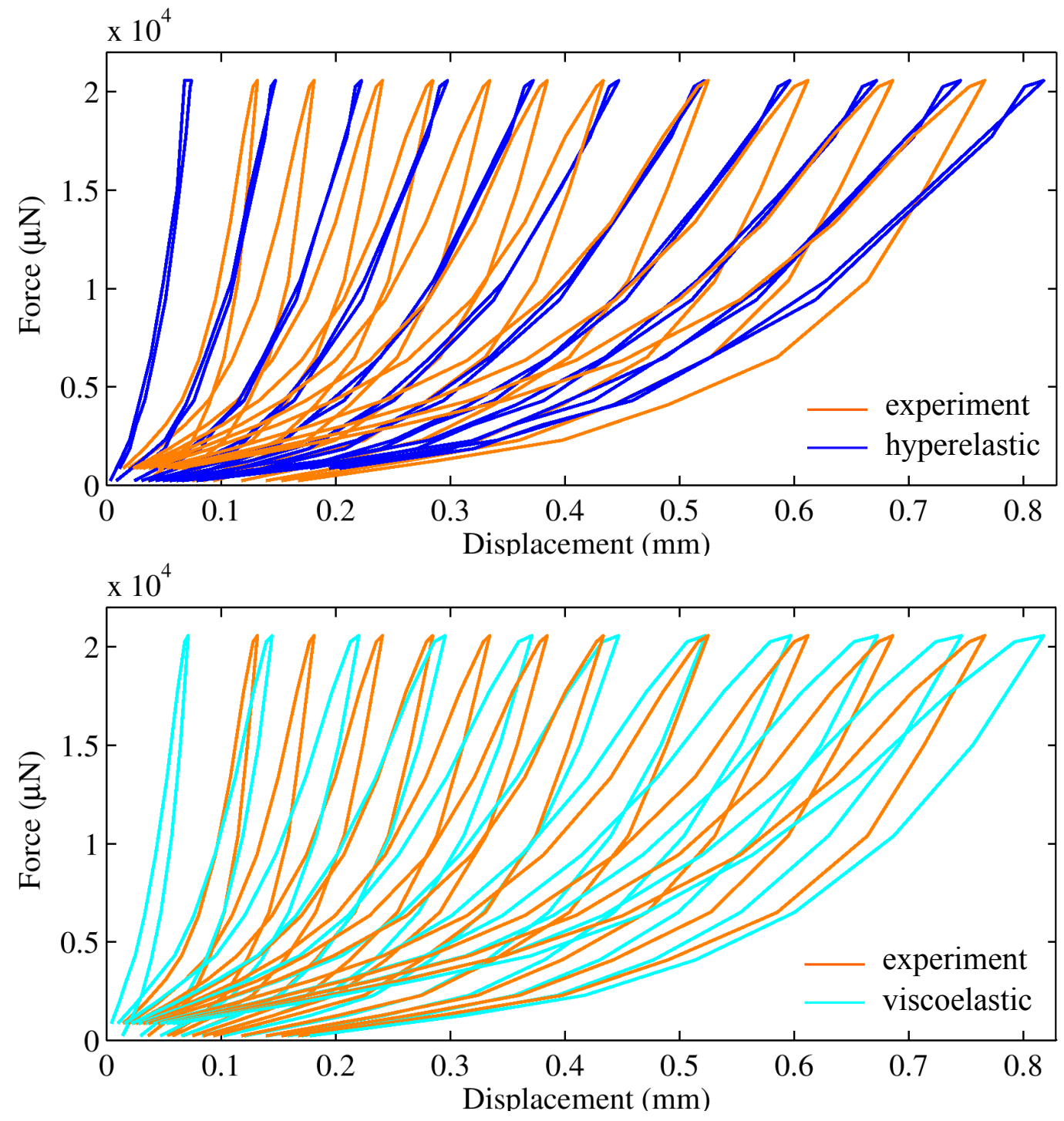

Figure 14: Comparison of the force versus displacement curve along axis $x$ for one sample of rat $\mathrm{G}$ and for several nodes: experimental, computed with a hyperelastic constitutive law $B\left(\kappa=57.6 \mathrm{kPa}, C_{1}=1.36 \mathrm{kPa}\right.$ and $\left.C_{3}=71.9 \mathrm{kPa}\right)$ and with a visco-hyperelastic law $E\left(\kappa=62.4 \mathrm{kPa}, C_{1}=2.78 \mathrm{kPa}, C_{3}=41.8 \mathrm{kPa}, \tau=0.293\right)$. The hyperelastic curve has been smoothed not to represent some artifacts created by vibrations. The experimental displacement is computed by image registration. 
Fig. 15 displays all the results obtained by identification of the parameters of the hyperelastic potentials and of the corresponding viscoelastic laws. We can see the significant increase in accuracy obtained with the viscoelastic laws, especially when the frequency decreases. Mann-Whitney post-processing of the error values reveals that adding a viscous contribution significantly increases the quality of the identification; the p-value is below 0.0001 for all couples of laws (A, B, C, K, L vs. D, E, F, L, M respectively).

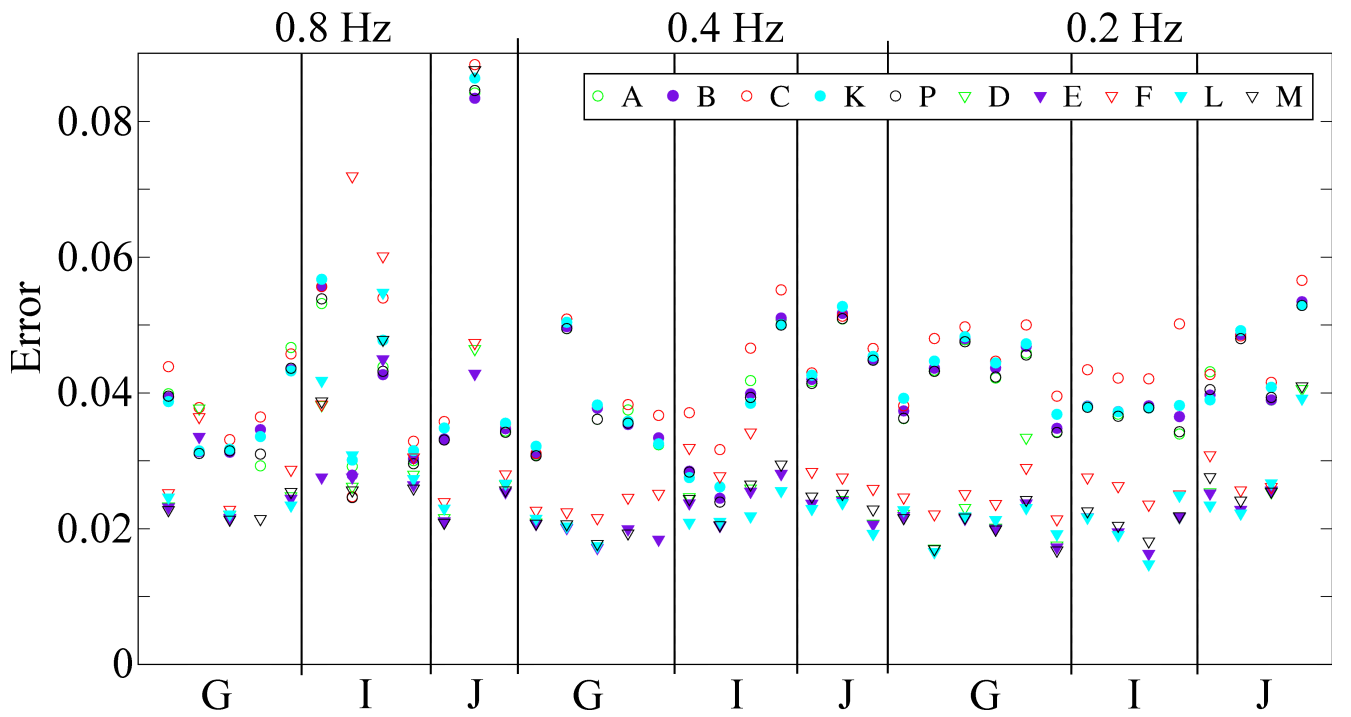

Figure 15: Distribution of the error obtained for all the samples for the identified hyperelastic potentials, with and without a viscous contribution. Laws D, E, F, L and M (triangles) are viscoelastic laws that correspond to the hyperelastic potentials A, B, C, K, $\mathrm{P}$ (circles) modified with a viscous contribution. X-axis: G, I, J are the three tested rats. Top: tested frequencies.

Fig. 16 displays the identification results for the viscoelastic laws for the tested frequencies. Altogether, the performance of law $\mathrm{F}$ is significantly lower than all the other laws (the p-factor is $\leq 0.05$ with respect to law $\mathrm{D}, \leq 0.001$ 
with respect to law $\mathrm{M}$ and $\leq 0.0001$ with respect to laws $\mathrm{E}$ and $\mathrm{L}$ ). Tab. 5 displays the identified values for the parameters of the viscoelastic laws. The parameters related to the elastic potentials $\left(\mathrm{C}_{i}, \kappa, \mathrm{k}_{i}\right)$ are averaged over all the samples and all the frequencies as the influence of the frequency is negligible. The obtained values are actually similar to those obtained with the purely elastic identification (Tab. 4) which makes sense as the latter values are the initial guess of the viscoelastic identification. However, $\tau$ significantly increases when the frequency decreases $(\mathrm{p} \leq 0.0001)$; its values are therefore given per frequency.

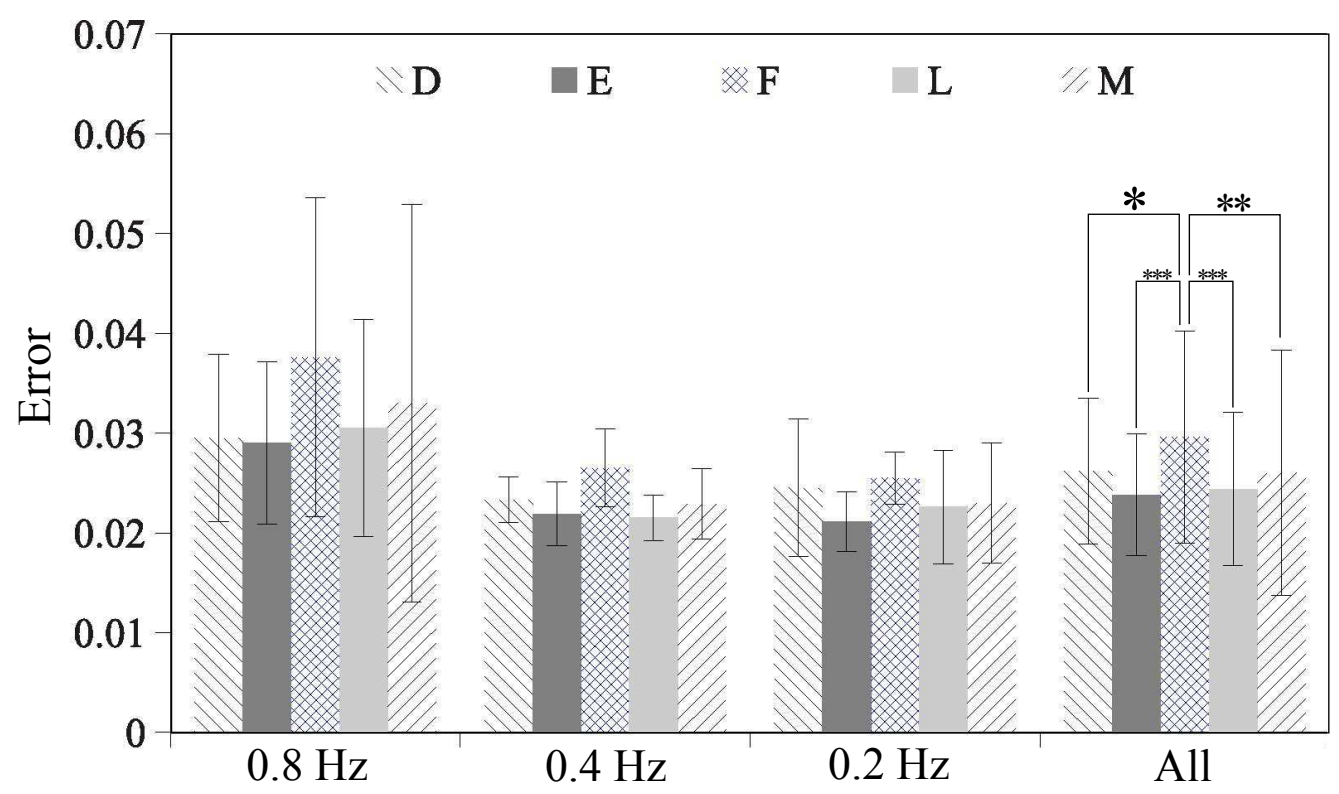

Figure 16: Comparison of the performance of different forms of viscoelastic potentials for various frequencies, among all the tested samples $(12$ at $0.8 \mathrm{~Hz}, 12$ at $0.4 \mathrm{~Hz}, 14$ at 0.2 $\mathrm{Hz}$, all from three different rats). ${ }^{*}$ corresponds to $\mathrm{p} \leq 0.05,{ }^{*}$ corresponds to $\mathrm{p} \leq 0.001$, $* * *$ corresponds to $\mathrm{p} \leq 0.0001$. 


\begin{tabular}{|c|c|c|c|}
\hline Law & Parameter & Value & Unit \\
\hline & $\kappa$ & $7.06 \mathrm{e} 4 \pm 2.93 \mathrm{e} 4$ & $\mathrm{~Pa}$ \\
\hline $\mathrm{D}$ & $C_{1}$ & $3.22 \mathrm{e} 3 \pm 9.53 \mathrm{e} 2$ & $\mathrm{~Pa}$ \\
& $C_{2}$ & $2.04 \mathrm{e} 3 \pm 2.86 \mathrm{e} 3$ & $\mathrm{~Pa}$ \\
& $C_{3}$ & $2.92 \mathrm{e} 4 \pm 1.52 \mathrm{e} 4$ & $\mathrm{~Pa}$ \\
\hline $\mathrm{E}$ & $C_{1}$ & $3.54 \mathrm{e} 3 \pm 9.48 \mathrm{e} 2$ & $\mathrm{~Pa}$ \\
& $C_{3}$ & $3.30 \mathrm{e} 4 \pm 1.58 \mathrm{e} 4$ & $\mathrm{~Pa}$ \\
\hline $\mathrm{L}$ & $C_{1}$ & $4.04 \mathrm{e} 3 \pm 1.07 \mathrm{e} 3$ & $\mathrm{~Pa}$ \\
& $C_{4}$ & $9.62 \mathrm{e} 4 \pm 5.09 \mathrm{e} 4$ & $\mathrm{~Pa}$ \\
\hline $\mathrm{M}$ & $k_{1}$ & $5.58 \mathrm{e} 3 \pm 1.69 \mathrm{e} 3$ & $\mathrm{~Pa}$ \\
& $k_{2}$ & $4.86 \pm 1.42$ & - \\
\hline
\end{tabular}

\begin{tabular}{|c|c|c|c|}
\hline Frequency & Parameter & Value & Unit \\
\hline $0.8 \mathrm{~Hz}$ & & $5.06 \mathrm{e}-2 \pm 2.53 \mathrm{e}-2$ & \\
$0.4 \mathrm{~Hz}$ & $\tau$ & $1.23 \mathrm{e}-1 \pm 3.87 \mathrm{e}-2$ & $\mathrm{~s}$ \\
$0.2 \mathrm{~Hz}$ & & $2.57 \mathrm{e}-1 \pm 4.22 \mathrm{e}-2$ & \\
\hline
\end{tabular}

Table 5: Identified parameters for viscoelastic potentials. 


\section{Discussion}

This paper presents a method to determine material parameters for the lung parenchyma using advanced methods from both the experimental and the theoretical point of view. Thanks to this method, we obtain a reliable viscoelastic constitutive law that is suitable for Finite Element calculations.

The experimental protocol has been improved since (Rausch et al., 2011). Measuring the thickness of each sample individually revealed its non-negligible variability throughout the set of samples; this distribution is now taken into account in the simulation. The other main improvement is the use of an optical method to determine the displacement field at the sample surface. It is worth noticing that as the samples are really thin, we can consider that this surface information is sufficient to describe the behavior of the tissue. In our case, knowing the displacement field is of high importance as we are particularly interested in the lateral contraction of the sample. When we optically check the results of the image registration by superimposing the image and the corresponding mesh (Fig. 8 for instance), we can see that this optical method applied to the natural pattern of the parenchyma gives fully satisfactory results, despite some light reflections at the sample surface. These artifacts are indeed smoothed by the Finite-Element kinematics inherent to the image registration method.

The theoretical constitutive framework used in this paper is adapted from an existing model proposed in (Holzapfel and Gasser, 2001). It allows the description of the non-linear elasticity as well as the viscosity for both the isochoric and the volumetric components of the deformation, not only the isochoric one. 
In the present work, a quantitative determination of elastic as well as viscous properties of rat lung parenchyma is provided. The order of magnitude of the strain in our protocol is 0 to $45 \%$. In this range, the non-linear elastic behaviour of lung parenchyma is equally well described by a polynomial potential of degree $\geq 3$ associated to a linear component (degree 1) or an exponential potential. No significant influence of the testing frequency was found in the identified elastic parameters. For the volumetric aspect of the law, we could determine that both Ogden and Sussman-Bathe give the same result and that the volumetric parameter $\kappa$ is rather accurately identified (c.f. low standard deviation). In (Rausch et al., 2011), values for the parameters for a law of type $B$ are given. $\mathrm{C}_{1}$ is similar to the one of this paper, while $\kappa$ and $\mathrm{C}_{3}$ are smaller. The explanation can come from a better description of the lateral contraction in the present paper, that highly influences the determination of $\kappa$, as well as the consideration of the actual thickness of the sample.

Adding a viscous component to the identified non-linear elastic constitutive law significantly increases the quality of the identification, especially at low frequency where the error is cut by half compared to the purely elastic case. It allows the description of the experimentally observed hysteresis loop. The elastic contribution of the law is rather insensitive to the frequency, but the time constant $\tau$ significantly decreases with increasing frequency. It shows that the lower the frequency, the higher the viscous effects.

The main limitation of this work is that the uniaxial tension loading mode does not correspond to the mainly volumetric deformations the tissue would be subjected to in reality. It is not guaranteed that the constitutive 
law determined in this paper for an isochoric-dominated loading would not be influenced by a volumetric-dominated loading. Identifying the volumetric contribution using the width evolution is however a first step towards an accurate estimation of the compressible behavior of the homogeneous lung parenchyma.

A possible improvement for the presented method would be to replace the two optimization processes (image registration and inverse analysis) by a single one. It would consist in improving the mechanical regularization in the image registration, currently purely elastic, by taking into account the actual material law that is to be identified. This type of mechanical/optical identification method is described for instance in (Réthoré, 2010) and could be implemented in our in-house code in a straightforward way.

The present experimental protocol and identification method are now to be applied for more biological tissues. Although they are particularly adapted to compressible material, they could also be used for any soft biological tissue, possibly requiring the addition of a random pattern on the surface of the sample. Future work is to investigate the viscoelastic properties of human lung parenchyma as well as parenchyma samples altered with ARDS. These data could then be included into the computational model of the respiratory system that is developed in our group.

Acknowledgement 1. Support by the German Science Foundation/Deutsche Forschungsgemeinschaft DFG (Wa 1521/9 and Uh 88/7) is gratefully acknowledged. 


\section{Appendix A. Computation of the elastic stress and elasticity ten-} sors

\section{Appendix A.1. Kinematic quantities}

The decoupled form of the potential requires expressing modified strain tensors. We define $\mathbf{F}$ as the deformation gradient and $J=\operatorname{det} \mathbf{F}$ is the volume change. The Cauchy-Green strain tensor is therefore $\mathbf{C}=\mathbf{F}^{T} \mathbf{F}$. In the case of a decoupled hyperelastic potential, we apply a volumetric/isochoric multiplicative split of the deformation.

$$
\begin{aligned}
\mathbf{F} & =J^{1 / 3} \overline{\mathbf{F}} \\
\mathbf{C} & =J^{2 / 3} \overline{\mathbf{C}}
\end{aligned}
$$

s. where $\overline{\mathbf{F}}$ and $\overline{\mathbf{C}}$ are the modified deformation gradient and Green-Lagrange tensor respectively, representing the isochoric part of the deformation. The strain invariants are defined as:

$$
\left\{\begin{array}{l}
I_{1}(\mathbf{C})=\operatorname{tr} \mathbf{C} \\
I_{2}(\mathbf{C})=\frac{1}{2}\left((\operatorname{tr} \mathbf{C})^{2}-\operatorname{tr} \mathbf{C}^{2}\right) \\
I_{3}(\mathbf{C})=\operatorname{det} \mathbf{C}
\end{array}\right.
$$

The modified invariants associated with the isochoric contribution are defined as :

$$
\begin{aligned}
& \bar{I}_{i}=J^{-2 / 3} I_{i} \text { for } i=1,3 \Rightarrow \bar{I}_{3}=1 \\
& \bar{I}_{2}=J^{-4 / 3} I_{2}
\end{aligned}
$$




$$
\psi\left(\mathbf{C}, \boldsymbol{\Gamma}_{\text {vol }}, \overline{\boldsymbol{\Gamma}}\right)=\psi_{\text {vol }}^{\infty}(J)+\gamma_{\text {vol }}\left(\boldsymbol{\Gamma}_{\text {vol }}\right)+\psi_{\text {iso }}^{\infty}\left(\bar{I}_{1}, \bar{I}_{2}\right)+\gamma_{i s o}(\overline{\boldsymbol{\Gamma}})
$$

For the next section, we define the following quantities:

$$
\begin{aligned}
\psi_{i} & =\frac{\partial \psi_{i s o}^{\infty}}{\partial \bar{I}_{i}}, i=1,2 \\
p & =\frac{\partial \psi_{v o l}^{\infty}}{\partial J} \\
\psi_{i j} & =\frac{\partial^{2} \psi_{i s o}^{\infty}}{\partial \bar{I}_{i} \partial \bar{I}_{j}}, \quad i, j=1,2
\end{aligned}
$$

Appendix A.3. Stress tensors

According to the work of (Holzapfel and Gasser, 2001), the elastic second Piola-Kirchhoff stress tensors are defined by: 


$$
\begin{aligned}
& \mathbf{S}_{v o l}^{\infty}=J p \mathbf{C}^{-1} \\
& \mathbf{S}_{i s o}^{\infty}=J^{-2 / 3} \mathbb{P}:\left[2 \psi_{1} \mathbf{I}+2 \psi_{2}\left(\bar{I}_{1} \mathbf{I}-\overline{\mathbf{C}}\right)\right]
\end{aligned}
$$

593

$$
\mathbb{C}_{v o l}^{\infty}=J \tilde{p} \mathbf{C}^{-1} \otimes \mathbf{C}^{-1}-2 J p \mathbf{C}^{-1} \odot \mathbf{C}^{-1}
$$

$$
\mathbb{C}_{i s o}^{\infty}=\sum_{i=1,2} \mathbb{C}_{i s o, i}^{\infty}
$$

where:

$$
\begin{gathered}
\tilde{p}=p+J \frac{\partial p}{\partial J} \\
\mathbb{C}_{i s o, i}^{\infty}=\mathbb{P}: \hat{\mathbb{C}}_{i}^{\infty}: \mathbb{P}^{T}+\frac{2}{3}\left(J^{2 / 3} \hat{\mathbf{S}}_{i}^{\infty}: \mathbf{C}\right) \tilde{\mathbb{P}} \\
-\frac{2}{3}\left(\mathbf{C}^{-1} \otimes \mathbf{S}_{i s o, i}^{\infty}+\mathbf{S}_{i s o, i}^{\infty} \otimes \mathbf{C}^{-1}\right)
\end{gathered}
$$

601

602

$$
\hat{\mathbb{C}}_{i}^{\infty}=4 J^{-4 / 3} \sum_{j=1,2}\left(\psi_{i j} \mathbf{D}_{i} \otimes \mathbf{D}_{j}+\psi_{i} \mathbb{D}_{i}\right)
$$

$$
\mathbf{D}_{i}=\frac{\partial \bar{I}_{i}}{\partial \bar{C}}, \quad \mathbb{D}_{i}=\frac{\partial \mathbf{D}_{i}}{\partial \bar{C}}
$$




$$
\tilde{\mathbb{P}}=\mathbf{C}^{-1} \odot \mathbf{C}^{-1}-\frac{1}{3} \mathbf{C}^{-1} \otimes \mathbf{C}^{-1}
$$

604

The viscous contribution can be calculated from the elastic contribution, see Appendix B.

Appendix B. Computation of the viscous stress and elasticity tensors

Appendix B.1. Fictitious stress tensor

To solve the differential equation (7), we apply the following method:

$$
\begin{array}{r}
\dot{\mathbf{Q}}+\frac{1}{\tau} \mathbf{Q}=\beta \dot{\mathbf{S}} \\
\Leftrightarrow \dot{\mathbf{Q}}-\beta \dot{\mathbf{S}}=-\frac{1}{\tau} \mathbf{Q}
\end{array}
$$

10 Once the elastic stress tensor $\mathbf{S}$ is computed at time step $n+1$, we use a Newton scheme to compute the fictitious stress tensor at step $n+1$, using the history of these tensors:

$$
\begin{aligned}
& (\mathbf{Q}-\beta \mathbf{S})_{n+1}=(\mathbf{Q}-\beta \mathbf{S})_{n} \\
& \quad+(1-\theta) \Delta t \underbrace{(\dot{\mathbf{Q}}-\beta \dot{\mathbf{S}})_{n}}_{-\frac{1}{\tau} \mathbf{Q}_{n}}+\theta \Delta t \underbrace{(\dot{\mathbf{Q}}-\beta \dot{\mathbf{S}})_{n+1}}_{-\frac{1}{\tau} \mathbf{Q}_{n+1}} \\
& \Leftrightarrow \mathbf{Q}_{n+1}=\lambda_{1}\left[\lambda_{2} \mathbf{Q}_{n}+\beta\left(\mathbf{S}_{n+1}-\mathbf{S}_{n}\right)\right]
\end{aligned}
$$


with

$$
\lambda_{1}=\frac{\tau}{\tau+\theta \Delta t}, \quad \lambda_{2}=1-\frac{(1-\theta) \Delta t}{\tau}
$$

Appendix B.2. Viscous elasticity tensor

As described in (Holzapfel and Gasser, 2001), the overall elasticity tensor at time step $n+1$ is calculated as the sum of the elastic tensor and the viscous tensor, which leads to:

$$
\mathbb{C}_{n+1}=(1+\delta)\left[\mathbb{C}_{v o l}^{\infty}+\sum_{i=1,2} \mathbb{C}_{i s o, i}^{\infty}\right]_{n+1}
$$

where $\delta=\beta \exp (-\Delta t / 2 \tau)$ as we consider the viscous constants $\tau$ and $\beta$ to be the same for isochoric and volumetric contributions.

Balzani, D., Neff, P., Schröder, J., Holzapfel, G., 2006. A polyconvex framework for soft biological tissues. adjustment to experimental data. International Journal of Solids and Structures 43, 6052-6070.

Delfino, A., Stergiopulos, N., Moore, J.E., Meister, J.J., 1997. Residual strain effects on the stress field in a thick wall finite element model of the human carotid bifurcation. Journal of Biomechanics 30, 777-786.

Dolhnikoff, M., Mauad, T., Ludwig, M.S., 1999. Extracellular matrix and oscillatory mechanics of rat lung parenchyma in bleomycin-induced fibrosis. American Journal of Respiratory and Critical Care Medicine 160, 17501757. 
Doll, S., Schweizerhof, K., 2000. On the development of volumetric strain energy functions. Journal of applied mechanics 67, 17-21.

Fischer, B., Modersitzki, J., 2008. Ill-posed medicine - an introduction to image registration. Inverse Problems 24, 034008.

Fung, Y., 1993. Biomechanics: Mechanical Properties of Living Tissues. Springer.

Gao, J., Huang, W., Yen, R.T., 2006. Mechanical properties of human lung parenchyma. Biomedical Sciences Instrumentation 42, 172-180.

Haber, E., Heldmann, S., Modersitzki, J., 2009. A framework for imagebased constrained registration with an application to local rigidity. Linear Algebra and its Applications 431, 459-470.

Haber, E., Modersitzki, J., 2006. A multilevel method for image registration. SIAM Journal on Scientific Computing 27, 1594-1607.

Holzapfel, G., Gasser, T., 2001. A viscoelastic model for fiber-reinforced composites at finite strains: Continuum basis, computational aspects and applications. Computer methods in Applied Mechanics and Engineering 190, 4379-4403.

Ingenito, E., Mark, L., Davison, B., 1994. Effects of acute lung injury on dynamic tissue properties. Journal of Applied Physiology 77, 2689-2697.

Kallet, R.H., Katz, J.A., 2003. Respiratory system mechanics in acute respiratory distress syndrome. Respiratory care clinics of North America 9, 297-319. 
Lester, H., Arridge, S.R., 1999. A survey of hierarchical non-linear medical image registration. Pattern recognition 32, 129-149.

Mann, H., Whitney, D., 1947. On a test of whether one of two random variables is stochastically larger than the other. The annals of mathematical statistics $18,50-60$.

Martin, C., Uhlig, S., Ullrich, V., 1996. Videomicroscopy of methacholineinduced contraction of individual airways in precision-cut lung slices. European Respiratory Journal 9, 2479-2487.

Mijailovich, S.M., Stamenovic, D., Brown, R., Leith, D.E., Fredberg, J.J., 1994. Dynamic moduli of rabbit lung tissue and pigeon ligamentum propatagiale undergoing uniaxial cyclic loading. Journal of Applied Physiology $76,773-782$.

Modersitzki, J., 2004. Numerical Methods for Image Registration (Numerical Mathematics and Scientific Computation). Oxford university press.

Navajas, D., Maksym, G.N., Bates, J.H., 1995. Dynamic viscoelastic nonlinearity of lung parenchymal tissue. Journal of Applied Physiology 79, $348-356$

Ogden, R., 1974. On the overall moduli of non-linear elastic composite materials. Journal of the Mechanics and Physics of Solids 22, 541-553.

Pinart, M., Faffe, D., Sapiña, M., Romero, P., 2011. Dynamic nonlinearity of lung tissue: effects of strain amplitude and stress level. Journal of Applied Physiology 110, 653-660. 
Rausch, S., Martin, C., Bornemann, P., Uhlig, S., Wall, W., 2011. Material model of lung parenchyma based on living precision-cut lung slice testing. Journal of the Mechanical Behavior of Biomedical Materials 4, 583-592.

Rocco, P.R., Negri, E.M., Kurtz, P.M., Vasconcellos, F.P., Silva, G.H., Capelozzi, V.L., Romero, P.V., Zin, W.A., 2001. Lung tissue mechanics and extracellular matrix remodeling in acute lung injury. American Journal of Respiratory and Critical Care Medicine 164, 1067-1071.

Romero, P.V., Faffe, D.S., Cañete, C., 2011. Dynamic nonlinearity of lung tissue: frequency dependence and harmonic distortion. Journal of Applied Physiology 111, 420-426.

Réthoré, J., 2010. A fully integrated noise robust strategy for the identification of constitutive laws from digital images. International Journal for Numerical Methods in Engineering 84 (6), 631-660.

Suki, B., Bates, J., 2011. Lung tissue mechanics as an emergent phenomenon. Journal of Applied Physiology 110, 1111-1118.

Wall, W.A., Wiechert, L., Comerford, A., Rausch, S., 2010. Towards a comprehensive computational model for the respiratory system. International Journal for Numerical Methods in Biomedical Engineering 26, 807-827.

Yeoh, O., 1993. Some forms of the strain energy function for rubber. Rubber Chemistry and Technology 66, 754-771.

Yuan, H., Ingenito, E., Suki, B., 1997. Dynamic properties of lung parenchyma: mechanical contributions of fiber network and interstitial cells. Journal of Applied Physiology 83, 1420-31, discussion 1418-9. 